\title{
iTRAQ-based quantitative proteomic of tobacco (Nicotiana tabacum L.) identified major host proteins involved in photosystems throughout the aging process
}

\section{Conglian He}

Yunnan Academy of Tobacco Agricultural Siences

\section{Shubin Sun}

Research Center of Hubei Tobacco Industrial Co., Ltd of China

\section{Yun Tang}

Research Center of Hubei Tobacco Industrial Co., Ltd of China

Chenggang He

Yunnan Agricultural University

\section{Pengfei Li}

Research Center of Yunnan Aromatic Tobacco Company

Tianyang $\mathrm{Xu}$

Research Center of Yunnan Aromatic Tobacco Company

\section{Gaokun Zhao}

Yunnan Academy of Tobacco Agricultural Sciences

\section{Congming Zou}

Yunnan Academy of Tobacco Agricultural Sciences

\section{Zhonglong Lin}

Yunnan Academy of Tobacco Agricultural Sciences

\section{Yi Chen ( $\nabla$ cytobacco007@sina.com )}

Yunnan Academy of Tobacco Agricultural Sciences https://orcid.org/0000-0003-3828-8559

\section{Research article}

Keywords: iTRAQ, tobacco leaves, senescence, Proteomics, Photosynthesis

Posted Date: April 8th, 2020

DOI: https://doi.org/10.21203/rs.2.20550/v3

License: (9) This work is licensed under a Creative Commons Attribution 4.0 International License. Read Full License 
Page 2/32 


\section{Abstract}

Background: Leaf senescence is one of the most common manifestations in plant senescence and has an important effect on plant photosynthesis. Delaying or shorten the senescence of tobacco leaves is one of the important approaches to improve tobacco breeding. However, the molecular regulatory mechanism of tobacco leaf senescence in response to photosynthesis is still poorly understood. To gain insights into the senescence of tobacco leaves, we integrated analyses including photosynthesis, organelle ultrastructure, and proteome of tobacco leaves during senescence.

Results: The photosynthetic rate, intercellular $\mathrm{CO}_{2}$ concentration, stomatal conductance and transpiration rate showed a downward trend, and the stability of organelle decreased with the senescence of tobacco leaves. Isobaric Tag for Relative Absolute Quantitation (iTRAQ) and Parallel Reaction Monitoring (PRM) were used to analyze the proteins expressed in different periods that were estimate based on photosynthetic physiology and ultramicroscopic observations. A total of 321, 319, 223 differentially expressed proteins (DEPs) were identified from over maturity (OM) vs immature (IM), OM vs well maturity (WM) and WM vs IM, respectively, including 122/199, 124/195 and 125/98 up/down proteins, respectively. Kyoto Encyclopedia of Genes and Genomes (KEGG) analysis revealed that the DEPs were significantly enriched in metabolic pathways, biosynthesis of secondary metabolites, microbial metabolism in diverse environments and starch and sucrose metabolism. In addition, the down-regulated proteins were also involved in metabolic pathways, such as carbon sequestration of photosynthetic organisms and photosynthesis. PRM analysis was performed and indicated that ITRAQ is highly reliable in the identification of major proteins involved in tobacco senescence.

Conclusions: This study provided important technical references for screening of host photosynthetic proteins during tobacco leaves senescence and established a basis for scientifically understanding of the senescence mechanism of tobacco leaves.

\section{Background}

Leaf senescence is one of the most common manifestations of plant senescence [1,2], and plant senescence is regulated and affected by different genes and environments $[3,4,5,6]$. In gene regulation, gene rearrangement is a major mechanism to control leaf senescence. In addition, transcription factor activity and epigenetic mechanisms, including chromatin remodeling, DNA modification, and RNAmediated control of transcription factors and other senescence-associated genes is another key mechanism for regulation of gene expression during leaf senescence [7, 8]. In environment regulation, insufficient water and nutrient supply are the main causes of plant leaf senescence. In addition, abiotic stress, such as extreme temperature, drought, ozone, nutritional deficiency, pathogen infection, injury and shading, would also cause plant leaf senescence $[9,10,11]$.

During leaf senescence, chlorophyll degradation is one of the most important catabolism that will cause leaf yellowing during plant senescence. Simultaneously, large molecules such as proteins and nucleic 
acids are degraded, which causes a decline in photosynthesis during leaf senescence. Chlorophyll content is often used to characterize plant senescence $[12,13]$. Proteomics is an important part of systems biology, which is widely used to study protein expressions in plants and animals during their growth, development, mutation, and in changing external environments. With the development of proteomics, ITRAQ technology seems provide a reasonable way to study the photosynthesis of leaf senescence.

Tobacco is one of the classic model plants. During the cultivation process of flue-cured tobacco, there are technical measures of topping (removal of flowers at the top of plants) and wiping off the branches, that will change the pattern of material accumulation in the leaves and change the normal senescence pattern of tobacco leaves $[14,15]$. In the harvest process, the maturity of tobacco leaves is generally judged based on their appearance characteristics including color, fluff, and veins. However, these characteristics are often greatly affected by the subjective influence of harvesters, thus bring major defects in determination of the maturity of tobacco leaves [16]. The quality difference in fresh tobacco leaves will give rise to problems, in some case, leading to difficulty in curing and poor quality after curing of the flue-cured tobacco leaves. Therefore, clarifying the senescence mechanisms in tobacco leaves might have certain guiding significance for similar studies in other vegetable crops.

With the continuous improvement of proteomics technology, proteomics has been gradually applied to researches in tobacco. Das et al. used iTRAQ technology to compare the protein expressions on TMV infected and mock infected tobacco plants [17]. In that research, a total of 407 differential proteins were identified, and several major host proteins responsive to TMV infection/ replication were found. Most of the tobacco proteomics researches were involved in resistance to stress, pests and diseases [18, 19]. However, proteomics studies regarding to protein changes during the senescence of tobacco leaves, especially those related to photosynthesis, are scarce. Therefore, elucidating the molecular mechanism of photosynthesis response to senescence of tobacco leaves and screening host proteins involved in photosynthesis during the senescence process are of great significance, at least for accurate judgment of senescence in tobacco and other plants.

Proteomics can screen potential protein targets from differentially expressed proteins [20,21]. Highthroughput and high-resolution protein isolation and identification techniques are now commonly used for quantitative and qualitative analysis of proteins [22]. The iTRAQ for protein quantitative analysis has the characteristics of high sensitivity, strong separation ability, wide application range, high throughput, reliable results and high degree of automation [23]. The targeted proteomic technologies, such as SRM and PRM, were useful in quantification of selected proteins or peptides to verify specific scientific hypotheses. Compared with the traditional SRM technology, the parallel reaction monitoring (PRM) technology has the advantage of high sensitivity and good specificity [24, 25].

The purpose of this study was to screen potential senescence target proteins of tobacco represented by K326 cultivar by using iTRAQ technology. PRM technology was used to verify the expression of DEPs to identify potential target proteins associated with tobacco senescence and to verify the reliability of iTRAQ 
results. This study hypothesized that photosynthetic-related proteins exhibited significant expression levels during three senescence stages (IM, WM and OM) of tobacco leaves. In the aspect of proteomics, this study expanded our scientifically understanding of the senescence mechanism of tobacco leaves, and provided a theoretical basis for tobacco breeding since the finding of target proteins associated with senescence might has the potential to be exploited as target or marker to delay or shorten the senescence of tobacco leaves.

\section{Results}

\subsection{Photosynthesis at Different Maturity Stages}

During tobacco senescence, photosynthesis of tobacco leaves at three different maturity stages was analyzed. The photosynthetic rate at IM, WM and OM were $15.00 \mu \mathrm{mol} \cdot \mathrm{m}^{-2} \cdot \mathrm{s}^{-2}, 11.50 \mu \mathrm{mol} \cdot \mathrm{m}^{-2} \cdot \mathrm{s}^{-2}$ and $5.90 \mu \mathrm{mol} \cdot \mathrm{m}^{-2} \cdot \mathrm{s}^{-2}$, respectively. The photosynthetic rate of tobacco leaves at IM and WM was significantly higher than that at $\mathrm{OM}$, and there was no significant difference between IM stage and WM stage (Fig. 1A). The concentration of intercellular $\mathrm{CO}_{2}$ in tobacco leaves at IM, WM and OM were $510 \mu \mathrm{mol} \cdot \mathrm{mol}^{-1}, 400$ $\mu \mathrm{mol} \cdot \mathrm{mol}^{-1}$ and $280 \mu \mathrm{mol} \cdot \mathrm{mol}^{-1}$, respectively. The intercellular $\mathrm{CO}_{2}$ concentration of tobacco leaves in IM and WM periods was significantly higher than that in OM periods, and there was no significant difference between IM and WM periods (Fig. 1B). The stomatal conductance of tobacco leaves at IM, WM and OM were $0.24 \mathrm{mmol} \cdot \mathrm{m}^{-2} \cdot \mathrm{s}^{-1}, 0.13 \mathrm{mmol} \cdot \mathrm{m}^{-2} \cdot \mathrm{s}^{-1}$ and $0.11 \mathrm{mmol} \cdot \mathrm{m}^{-2} \cdot \mathrm{s}^{-1}$, respectively. The stomatal conductance of tobacco leaves in IM was significantly higher than that in WM and OM, and there was no significant difference between WM and OM (Fig. 1C). The stomatal conductance of tobacco leaves at IM, WM and OM was $2.75 \mathrm{mmol} \cdot \mathrm{m}^{-2} \cdot \mathrm{s}^{-1}, 1.40 \mathrm{mmol} \cdot \mathrm{m}^{-2} \cdot \mathrm{s}^{-1}$ and $1.30 \mathrm{mmol} \cdot \mathrm{m}^{-2} \cdot \mathrm{s}^{-1}$, respectively. The transpiration rate of tobacco leaves in IM was significantly higher than that in WM and $\mathrm{OM}$, and there was no significant difference between WM and OM (Fig. 1D). To sum up, photosynthetic rate, intercellular $\mathrm{CO}_{2}$ concentration, stomatal conductance and transpiration rate decreased with advancement of maturity stages.

\subsection{Chloroplasts configuration and ultrastructurey}

By observing the configuration and ultrastructure of chloroplasts, it was found that the IM leaves had orderly arrangement of chloroplasts, and complete chloroplast membrane and less starch granules than WM and OM (Fig. 2A). After enlargement, the proportion of starch granules in chloroplasts was small, and the chloroplast structure was complete, the cell wall structure was incomplete, and the grana lamellae were closely arranged (Fig. 2B). The structure of chloroplasts in WM leaves was similar to that in IM, in addition, starch granules were clearly visible, and the number of starch granules increased obviously and the volume increased in WM leaves (Fig. 2C). After enlargement, the arrangement of grana lamellae was loose, the gap was enlarged, and the structure of cell wall was clear, and the structure of chloroplast membrane was complete (Fig. 2D). During the OM stage, the number of chloroplasts decreased 
significantly, and the chloroplast membrane dissolved gradually, the volume and the number of starch granules were increased. With the dissolution of chloroplast membrane, the chloroplasts were exposed to cells (Fig. 2E). After amplification, almost all chloroplasts contained starch granules, the number of grana lamellae decreased compared with the WM and cell wall becomes obscured by lysisd (Fig. 2F). Thus, with the increasing of leaf age, the number of chloroplasts decreased, and the grana lamella became loose gradually from compact arrangement. The number of grana lamellae increased firstly and then decreased. The chloroplast membrane also gradually dissolved. The proportion of starch granules was positively correlated with increasing of leaf age.

\subsection{Identification of Proteins at Different Maturity Stages}

The number of secondary spectra and the number of resolved secondary spectra of all samples were 435604 and 158095 , respectively. The identification rate of spectra was more than $36.29 \%$. The number of identified peptide segments was 23346 , and the number of identified proteins was 4747 . The number of identified proteins containing at least two specific peptide segments was 3292 , which accounted for $69.35 \%$ of total protein amount.

\subsection{Quantitative Analysis of Protein between Samples}

If there is no significant change in the amount of the same protein between the two samples in relative quantification, the protein abundance ratio is close to 1. Pairwise comparisons of proteins with $p$-values < 0.05 and fold-changes $>1.2$ or $<0.83$ in abundance were regarded as differentially regulated proteins (DRPs). We identified a total of 321 DRPs, including 122 up-regulated and 199 down-regulated proteins, by comparing the OM against the IM (Fig. 3A). In contrast, we obtained 319 DRPs, including 124 upregulated and 195 down-regulated proteins, by comparing the OM against the WM (Fig. 3B). There were 223 DRPs, including 125 up-regulated and 98 down-regulated proteins between WM and IM (Fig. 3C). Fig. 4 was a Venn diagram showing significant differences in protein expression between pairwise comparisons. Comparisons of different proteins between the control groups revealed that there were 86 differentially expressed proteins among $\mathrm{OM}$ vs IM, OM vs WM and WM vs IM of the three control groups.

\subsection{KEGG Metabolic Pathway of Differentially Expressed Proteins}

To further investigate the biological functions of these DEPs, KEGG pathway analysis was performed using the BLAST v2.2.26 (NCBI) program. Four pathways were significantly enriched among the DEPs of OM vs IM, OM vs WM and WM vs IM, including "metabolic pathways", "Biosynthesis of secondary metabolites", "Microbial metabolism in diverse environments" and "Starch and sucrose metabolism". 
Comparing OM against IM, a total of 237 DEPs (74\%) were mapped to 79 pathways in the KEGG database (Table S1). Figure 5 (A) was a statistical pie chart of Pathway function, which ranked the top ten in the list of up and down-regulation differential proteins between over-mature (OM) and immature (IM) tobacco leaves. As can be seen from the figure, Pathway metabolic function types in the top 10 were different in all up-regulation and down-regulation differential proteins. The Pathway functional annotations of the two groups have the same four functions in the top 10; We identified 123 proteins (30 proteins were up-regulated and 93 proteins were down-regulated) that belong to "metabolic pathways", 55 proteins (22 proteins were up-regulated and 33 proteins were down-regulated) that belong to "Biosynthesis of secondary metabolites", 40 proteins (8 proteins were up-regulated and 32 proteins were down-regulated) that belong to "Microbial metabolism in diverse environments", 14 proteins (7 proteins were up-regulated and 7 proteins were down-regulated) that belong to "Starch and sucrose metabolism". In addition, up-regulated proteins were also involved in metabolic pathways such as "Protein processing in endoplasmic reticulum" (8), "Tyrosine metabolism" (7), "Phenylpropanoid biosynthesis" (6), "ABC transporters" (5) and "Plant-pathogen interaction" (5) and "Isoquinoline alkaloid biosynthesis" (5). Downregulation of protein involves in "Photosynthesis" (26), "Carbon fixation in photosynthetic organisms" (18), "Glycolysis/glycogenesis" (11), "Ribosome” (8), "Oxidative phosphorylation” (7), "Pentose phosphate pathway" (7) and so on (Fig. 5A).

Comparing OM against WM, a total of 237 DEPs (74\%) were mapped to 82 pathways in the KEGG database (Table S2). We identified 126 proteins (34 proteins were up-regulated and 92 proteins were down-regulated) that belong to "metabolic pathways", 64 proteins (28 proteins were up-regulated and 36 proteins were down-regulated) that belong to "Biosynthesis of secondary metabolites", 37 proteins (8 proteins were up-regulated and 29 proteins were down-regulated) that belong to "Microbial metabolism in diverse environments", 19 proteins (8 proteins were up-regulated and 11 proteins were down-regulated) that belong to "Starch and sucrose metabolism". Up-regulated proteins were also involved in "Phenylpropanoid biosynthesis" (8), "Tyrosine metabolism" (8), "Phenylalanine metabolism" (7), "Isoquinoline alkaloid biosynthesis" (6), "Splicer" (6) and "Protein processing in endoplasmic reticulum" (9), which were different from the functional metabolic pathways of down-regulated proteins. Downregulated proteins were involved in metabolic pathways, including "Photosynthesis" (25), "Carbon fixation in photosynthetic organisms" (13), "Glycolysis/glycogenesis" (9), "Pentose phosphate pathway" (8), "Glutathione metabolism" (7) and "Oxidative phosphorylation" (7) and so on (Fig. 5B).

Comparing WM against IM, a total of 163 DEPs (73\%) were mapped to 78 pathways in the KEGG database (Table S3). We identified 79 proteins (39 proteins were up-regulated and 40 proteins were downregulated) that belong to "metabolic pathways", 45 proteins (27 proteins were up-regulated and 18 proteins were down-regulated) that belong to "Biosynthesis of secondary metabolites", 26 proteins (10 proteins were up-regulated and 16 proteins were down-regulated) that belong to "Microbial metabolism in diverse environment", 12 proteins (7 proteins were up-regulated and 5 proteins were down-regulated) that belong to "Starch and sucrose metabolism". In addition, up-regulated proteins were also involved in metabolic pathways including "Phenylpropanoid biosynthesis" (6), "Protein processing in endoplasmic reticulum" (6), "Phenylalanine metabolism” (5), "Plant-pathogen interaction” (5), "ABC protein 
transporters" (4) and "Glutathione metabolism" (4). Down-regulated proteins were involved in metabolic pathways including "Carbon fixation in photosynthetic organisms" (12), "Ribosomes" (8),

"Photosynthesis" (7), "Glycolysis/gluconeogenesis" (5), "Pentose phosphate pathway" (5) and "Fructose and mannose metabolism" (4) and so on (Fig. 5C).

\subsection{Change in abundance of differential proteins}

A total of 86 DEPs from six significantly enriched pathways in the senescence of tobacco leaves are shown with their abundance patterns and functional annotation in Fig. 6. Many DEPs involved in metabolic pathways (32), biosynthesis of secondary metabolites (15), metabolism of microorganisms in different environments (11), carbon sequestration of photosynthetic organisms (10), and some DEPs involved in photosynthesis (8), starch and sucrose metabolism (6) were reduced sharply. In photosynthesis metabolic pathways, there were eight proteins that related to photosynthesis, including A0A140G1P2, A0A1S4DCM1, A0A140G1X0, D2K7Z2, A0A140G1R2, A0A1S4CFV4, A0A140G1P8 and A0A140G1S1.

\subsection{Photosynthetic proteins expression patterns}

Photosynthesis is critical for tobacco leaves yield and quality; hence, we investigated the abundance of proteins involved in photosynthesis pathway. Several DEPs concentrated in photosynthetic pathways and one typical pathway was shown in Fig. 7A. Almost all the photosynthetic DEPs enriched in core parts of photosynthesis, such as photosystems (I and II), cytochrome b6f complex, electron transports, and ATP synthases. Compared OM with IM, there were 23 DEPs involved in photosynthetic pathway, including one up-regulated protein and 22 down-regulated proteins. 24 DEPs involved in photosynthetic pathway were shown in Fig.7 (B) by comparing OM with WM, including one up-regulated protein and 23 down-regulated proteins. The enrichment of all photosynthetic DEPs was similar to that in OM vs IM. Compared WM with $I M$, there were 9 DEPs involved in photosynthetic pathway, including 2 up-regulated proteins and 7 downregulated proteins which enriched in photosystems (I and II) and ATP synthases (Fig. 7C).

\subsection{PRM verification}

Three proteins, including A0A1S4DCM1, A0A140G1R2 and A0A140G1P2 which were significantly expressed, and proteins related to photosynthesis were selected and their expression was examined by PRM. Skyline can not only visualize the target peptide ion signal which is collected by mass spectrometry, but also judge the quality and content of the peptide signal by observing the peak shape, peak intensity, and retention time consistency [26]. The effect is immediately visible when the ratios are presented in a density plot (Fig. 8A, B and C) or in a PRM plot (Fig.9 A, B and C). Compared with the quantitative results of iTRAQ, three differentially expressed proteins showed the same level (A0A1S4DCM1 was up-regulated 
and A0A140G1R2 and A0A140G1P2 were down-regulated) of expression which confirmed the reliability of iTRAQ.

\section{Discussion}

Photosynthetic rate, intercellular $\mathrm{CO}_{2}$ concentration, stomatal conductance and transpiration rate are comprehensive evaluation indicators of photosynthesis [27]. Photosynthetic rate and intercellular $\mathrm{CO}_{2}$ concentration in IM and WM were significantly higher than those in OM. Canales et al. cultured sunflower in different concentrations of $\mathrm{CO}_{2}$, and found that sunflower senescence was delayed in high concentrations of $\mathrm{CO}_{2}$ [28]. There were researches indicated that higher intercellular $\mathrm{CO}_{2}$ concentration and stomatal conductance were factors that improved photosynthesis. During the senescence of tobacco leaves, the decrease of stomatal conductance resulted in the decrease of gas exchange, which decreased the intercellular $\mathrm{CO}_{2}$ concentration and transpiration rate, then resulted in the decrease of photosynthetic rate. The decline of photosynthesis related index indicated that the photosynthesis of tobacco leaves decreased significantly during senescence.

The number of chloroplasts showed a downward trend with the increasing of tobacco leaf age. The grana lamellae gradually became loose from close arrangement, and its amount increased first and then decreased, and the chloroplast membrane gradually dissolved. The proportion of starch grains was positively correlated with the increasing of tobacco leaf age, which was consistent with the results of Mayta et al. [29]. There are lots of studies found that sugar was an important signal for regulating plant metabolism and development. The accumulation of sugar in leaves can lead to plant senescence [30]. Starch granules would be accumulated during the process of leaves growth and the chloroplast structure would be broken down when starch granules were accumulated enough [31]. The breaking down of chloroplast structure would weaken the photosynthesis of leaves.

To maximize information on the tobacco proteome, we prepared and identified protein extracts from three stages of tobacco leaves using iTRAQ coupled with LC-MS/MS technology. There were differences among the pairwise comparisons in protein expression, which indicated that the divergences in the appearance of tobacco leaves at various stages were accompanied by protein expression differences. Between OM vs IM and OM vs WM, the number of down-regulated proteins was greater than that of upregulated proteins, which indicated that the number of proteins decreased during tobacco leaves senescence. In the senescence of tobacco leaves, up-regulation proteins may be involved in the material catabolic pathway, while down-regulation proteins were mainly involved in substance anabolic pathway and the metabolic process related to photosynthesis. Zhang et al. [32] found that up-regulated proteins were involved in energy metabolism, and down-regulated proteins were involved in photosynthesis and electron transport by proteomic analysis of rice aging process, which is similar to the results of this study.

KEGG metabolic pathways analysis of differential proteins was conducted to further understand main biochemical metabolic pathways and signal transduction pathways that DEPs involved in. The DEPs involved in "Metabolic pathways", "Biosynthesis of secondary metabolites" and "Microbial metabolism in 
diverse environments", "Starch and sucrose metabolism". Ma et al. [33] carried out proteomic studies on four developmental stages of daylily flower. KEGG annotation results showed that DEPs were enriched in starch and sucrose metabolism. Starch accumulated slowly in the early stage and reached the peak at the mature stage. With the increasing of leaf age, starch gradually metabolized and its content showed a downward trend [34]. Secondary metabolism was the metabolic process of nicotine and major aroma precursor's synthesis and catabolism in plants. Studies have shown that the content of nicotine and reducing sugar in leaves increased with the increasing of tobacco leaf age [35]. "Microbial metabolism in diverse environments" comes up as a significantly enriched category may be associated with endophytes. Endophytes exist in plant tissues with the effects of promoting plant growth and development, participating in plant metabolic activities and regulating plant hormone signals [36, 37]. Studies have shown that both of the richness and diversity of endophytic fungi increase with the senescence of tobacco leaves [38]. The internal environment of the tobacco leaves changes with the increase of tobacco age, which results in the significant accumulation of differential proteins in "Microbial metabolism in diverse environments".

Up-regulated proteins were also involved in the metabolic pathways including "Endoplasmic reticulum protein processing" and "Phenylpropanoid biosynthesis". Phenylpropanoid was a kind of secondary metabolite, and its protein expression was up-regulated in the biosynthesis of phenylpropanoid, which further indicated that the biosynthesis process of secondary metabolite increases with the increasing of tobacco leaf age. During the senescence of tobacco leaves up-regulated proteins were involved in endoplasmic reticulum protein processing. Proteins processed by endoplasmic reticulum may due to protein degrading enzymes, which speed up the catabolism of proteins and other substances. Zhang et al. [39] found that proteases related to protein degradation, such as cysteine protease, aspartic protease and proteasome subunit alpha type-3, were up-regulated during tobacco leaves senescence, which indicated that the synthesis and catabolism of proteins were simultaneous in the senescence of tobacco leaves. There are many reasons for the reduction of protein during the senescence of tobacco leaves, including the decrease in the rate of protein synthesis, the acceleration of the degradation rate and the contact between the substrate and the hydrolase [40].

The proportion of down-regulated proteins in "Metabolic pathways" and "Microbial metabolism in different environments" was higher than that of up-regulated proteins. Metabolic pathways included anabolism and catabolism. The down-regulation of protein expression related to metabolic pathways and microbial metabolism in different environments indicated that the metabolic ability of tobacco leaves declined during tobacco senescence. Down-regulated proteins were also involved in metabolic pathways including "Photosynthesis", "Carbon fixation in photosynthetic organisms", "Glycolysis and Glycogenesis". There are two stages in Photosynthesis, including light reaction and dark reaction. During light reaction, leaves absorbed light energy to reduce NADP ${ }^{+}$to NADPH and phosphorylate ADP to ATP, which provided energy for carbon assimilation in dark reaction [41, 42, 43].The decline of protein expression involved in photosynthesis, carbon sequestration of photosynthetic organisms and glycolysis and glycogen regeneration indicated that photosynthesis and sugar synthesis were weakened during 
tobacco leaves senescence. Plaxton et al. [44] found that plant glycolytic enzymes were multifunctional proteins involved in the process, rather than carbohydrate metabolism. ATP was produced in glycolysis pathway [45], the down-regulation of protein expression related to glycolysis and glycogenesis indicated that ATP synthesis was decreased and sugar synthesis weakened during tobacco leaves senescence.

According to the enrichment of 86 proteins, they were mainly involved in metabolic pathways, including metabolic pathways, biosynthesis of secondary metabolites, metabolism of microorganisms in different environments, carbon sequestration of photosynthetic organisms, photosynthesis and metabolism of starch and sucrose. Photosystem I and II were photosynthetic electron transport centers [46]. According to the search results of 8 proteins related to photosynthesis, 3 proteins with significant differences between the comparison groups were screened for PRM verification. In the PRM validation process, three differentially expressed proteins were consistent with the quantitative results of iTRAQ in pairwise comparisons. Therefore, three differentially expressed proteins including A0A1S4DCM1, A0A140G1R2 and A0A140G1P2 were likely to be key regulators of tobacco leaves senescence, and can be used as marker protein for tobacco leaves senescence.

Photoreaction of photosynthesis is driven by protein complexes such as photosystem I, photosystem II, cytochrome b6/f complex, ATP synthase, and so on [47]. Oxygen-evolving enhancer protein 3-2, chloroplastic-like was a protein related to photosystem II, which participated in oxygen release during photosynthesis and maintained the stability of photosynthesis system $[48,49,50]$. In this study, the expression of A0A1S4DCM1 was up-regulated during tobacco leaves senescence, which was consistent with previous research results $[51,52,53]$. It was indicated that during the senescence of tobacco leaves, attributing to the decline of photosynthesis, the A0A1S4DCM1 is increased in order to maintain the stability of the photosynthetic system. In addition, oxygen-evolving enhancer proteins are products of degradation of the photosynthetic system, and the oxygen-evolving enhancer protein increased with the degradation of the photosynthetic system [54]. A0A140G1R2 (Photosystem I P700 chlorophyll an apoproteinA2) is an important protein for chloroplasts to maintain normal physiological functions [55, 56]. In the senescence of tobacco leaves, the decrease of A0A140G1R2 indicates that photosynthetic electron transport in senescent leaves is gradually inhibited, thus decreasing photosynthesis. A0A140G1P2 (Photosystem II protein D1) is the core subunit of photosystem II, which plays an important role in maintaining the stability of photoreaction center system II. It would also provide binding sites for electron-transfer oxidative coenzyme factors and involve in the electron transfer of most cofactors and pigment molecules [57]. The down-regulation of PSII protein D1 indicated that the transmission of electronic capabilities and the stability of PSII were decreased, and the function of PSII was weakened.

\section{Conclusion}

In this study, tobacco leaf photosynthetic rate, intercellular $\mathrm{CO}_{2}$ concentration, body surface conductivity, transpiration rate, and organelle ultrastructure all changed significantly. Combining proteomics technology and physiological changes for the first time, photosynthesis during tobacco senescence was 
described. Physiological changes revealed the mature metabolism of tobacco leaves; and dynamic protein changes obtained using ITRAQ revealed important metabolic pathways during tobacco senescence from the protein level, including "metabolic pathways", "biosynthesis of secondary metabolites", Metabolism in different environments "," starch and sucrose metabolism "and" photosynthesis "and so on. Finally, through PRM verification of photosynthesis-related differential expressed proteins, three differential expressed proteins, including A0A1S4DCM1, A0A140G1R2 and A0A140G1P2 were significant differences at three growth stages which would be target proteins or indicators for tobacco leaves senescence. All iTRAQ dataset related to this study has been public available on iProX (www.iprox.org) with ID IPX0001668000/ IPX0001668001/ IPX0001668002.

\section{Methods}

\subsection{Plant Materials}

The seeds of the tobacco variety K326 were acquired from Zhongyan Tobacco Seed Co., Ltd, China. Tobacco seedlings (Nicotiana tabacum L.) were transplanted to potting mixture. The seedlings were grown from April to August 2018 under typical Yunnan climate conditions. The middle leaves $\left(11^{\text {th }}\right.$ leaf position from the bottom) of tobacco plants from 3 periods, including IM (70 days after transplanting), WM (80 days after transplanting) and OM (90 days after transplanting) were separately collected, every stage had three replication of the experiment and then stored at $-80^{\circ} \mathrm{C}$ immediately until sent two replication to Wuhan Jinkairui Bioengineering Co., Ltd. for testing (Fig. 10).

\subsection{Determination of Photosynthetic Rate}

LI-6400 portable photosynthetic system (Li-Cor Inc., Lincoln, NE, USA) was used to measure the photosynthetic indexes of tobacco leaves at different maturity stages [58]. The photosynthetic rate, intercellular carbon dioxide concentration, stomatal conductance and transpiration rate of tobacco leaves were measured at IM, WM and OM stages, respectively. The determination time was once every two hours from 8:00 to 18:00 and five times in total. The observation data for statistical analysis was calculated by the average value. During the measurements, the leaf temperature was adjusted to $25^{\circ} \mathrm{C}$. Sigma Plot 12.3 (Systat Software Inc., Chicago, IL, USA) was used to plot according to the data that were presented as means \pm standard deviation (SD). One-way analysis of variance (ANOVA) was used to determine a significant difference between the groups with A 2-tailed $p$-value $<0.05$ was considered to be statistically significant.

\subsection{Electron Microscope Scanning}

This analysis was referred to Li et al.'s [59] methods with slight modification. Different maturity of fresh tobacco leaves were cut into $3 \mathrm{~mm} \times 3 \mathrm{~mm}$ size leaves, avoiding the main vein and large lateral vein of leaves. Then placed in a penicillin bottle and immersed the leaves in $4 \%$ glutaraldehyde fixing solution. 
The leaves were fully immersed in fixing solution by syringe extraction. The samples were fixed by $1 \%$ $\mathrm{OsO}_{4}$ (osmium tetroxide) for 2 hours and dehydrated by acetone. Then embedded the samples by epoxy resin (spi-812) for 24 hours and sliced (German Leica uc6) into thin slices. The slices were stained with lead citrate and uranyl acetate then observed and photographed the slices under Hitachi-A-2 transmission electron microscope.

\subsection{Protein extraction and digestion}

Middle tobacco leaves ( $11^{\text {th }}$ leaf position) of three periods were harvested and ground with a tissue homogenizer, in TissueLyzer LT (Qiagen) with liquid nitrogen until the leaves became fine powder. The ground powder was resuspended in lysis buffer (7 M urea, $2 \mathrm{M}$ thiourea, 4\% SDS (sodium salt), $40 \mathrm{mM}$ Tris-Cl, pH 8.5, 1 mM PMSF (phenylmethanesulfonyl fluoride), 2 mM EDTA (ethylene diamine tetraacetic acid)) for $5 \mathrm{~min}$. The suspension was added $10 \mathrm{mM}$ DL-Dithiothreitol (DTT) and placed in an ultrasonic cell disruptor (JY92-11N, Ningbo, China) sonicated for $15 \mathrm{~min}$ in an ice bath, then centrifuged at $13000 \mathrm{~g}$, $4{ }^{\circ} \mathrm{C}$ for 20 minutes in a desktop high speed refrigerated centrifuge (TGL-20M, Hunan, China). After that, transferred the supernatant to a new centrifugal tube, and added cold acetone with a volume of 4 times the supernatant to the centrifugal tube, which was placed overnight at $-20^{\circ} \mathrm{C}$.

Collecting the precipitation of protein by centrifugation then dried in air. Added $8 \mathrm{M}$ urea/100 mM Boranetriethylamine complex (TEAB, $\mathrm{pH}$ 8.0) solution to redissolve the protein. Then added DL-Dithiothreitol till the final concentration was $10 \mathrm{mM}$, and the reduction reaction was carried out in water bath at $56{ }^{\circ} \mathrm{C}$ for 30 minutes. After that, added lodoacetamide (IAM) till the final concentration was $55 \mathrm{mM}$ and the alkylation reaction was carried out at dark in $25^{\circ} \mathrm{C}$ for 30 minutes. The protein concentration was determined by Bradford method [60].

\subsection{Separation and Identification of Proteins}

Protein extraction and separation, ITRAQ quantification, bioinformatics analysis and PRM validation were dedicatedly carried out according to the following procedures (Fig. 11).

\subsubsection{Enzymatic Hydrolysis and Desalination}

$100 \mu \mathrm{g}$ of protein from each sample was taken for trypsin digestion and then diluted 5 times with 100 $\mathrm{mM}$ Triethylamine borane (TEAB). Trypsin was added at a mass ratio of 1:50 (trypsin: protein) and theenzymatic hydrolysis was digested overnight at $37^{\circ} \mathrm{C}$. After enzymatic hydrolysis, the peptide segments were desalted by AAA-C18 column ( $150 \mathrm{~mm} \times 4.6 \mathrm{~mm}$ I.D., $5 \mu \mathrm{m}, \mathrm{AB}$ SCIEX), and then frozen and dried in vacuum [61].

\subsection{2 iTRAQ labeling}


Dissolved the Peptides with 0.5M TEAB and labeled with iTRAQ-8 kit (AB SCIEX, USA) according to the manufacturer's instructions, Six samples including IM-1, IM-2, WM-1, WM-2, OM-1, OM-2 were labeled with iTRAQ tag 115, iTRAQ tag 116, iTRAQ tag 117, iTRAQ tag 118, iTRAQ tag 119 and iTRAQ tag 121, respectively. Get all of the labeled samples together and then fractionated the mixed samples with a highperformance liquid chromatography (HPLC) system (Thermo DINOEX Ultimate 3000 BioRS, USA) with a Durashell C18 $(5 \mu \mathrm{m}, 100 \AA, 4.6 \times 250 \mathrm{~mm})$. A total of 42 secondary fractions were collected and merged into 12 fractions for analysis. The fractions were then desalinated on the Strata-X C18 column (Phenomenex, Torrance, USA) and dried in vacuum.

\subsubsection{LC-MS/MS Analysis}

Referring to the method of Zhu et al. [62], LC-MS/MS analysis was performed on Triple TOF 5600 plus mass spectrometer (AB SCIEX, Massachusetts, USA). Dissolved the polypeptide samples in $2 \%$ acetonitrile/ $0.1 \%$ formic acid and analyzed by Triple TOF 5600 plus mass spectrometer coupled with Eksigent nanoLC system (SCIEX, USA). The polypeptide solution was added to the C18 capture column (5 $\mu \mathrm{m}, 100 \mu \mathrm{m} \times 20 \mathrm{~mm})$, and gradient elution was carried out on the C18 analytical column $(3 \mu \mathrm{m}, 75 \mu \mathrm{m} \times$ $150 \mathrm{~mm}$ ) at a 90 -minute time gradient and $300 \mathrm{~nL} / \mathrm{min}$ flow rate. The two mobile phases are respectively mobile phase $A\left(2 \%\right.$ acetonitrile/ $0.1 \%$ formic acid $/ 98 \% \mathrm{H}_{2} \mathrm{O}$ ) and mobile phase $B(98 \%$ acetonitrile $/ 0.1 \%$ formic acid/2\% $\mathrm{H}_{2} \mathrm{O}$ ). For information dependent acquisition (IDA), the first-order mass spectrogram was scanned with $250 \mathrm{~m} / \mathrm{s}$ ion accumulation time, and the second-order mass spectrogram of 30 precursor ions was collected with $50 \mathrm{~ms}$ ion accumulation time. The MS1 spectra was collected in the range of $350-1500 \mathrm{~m} / \mathrm{z}$, and the MS2 spectra was collected in the range of $100-1500 \mathrm{~m} / \mathrm{z}$. The precursor ions were excluded from reselection for $15 \mathrm{~s}$.

\subsubsection{Protein Identification and data analysis}

The original MS/MS data were submitted to ProteinPilot Software v4.5 (Applied Biosystem, USA) for data analysis. Combining ProteinPilot's Paragon ${ }^{\mathrm{TM}}$ database search algorithm (ProteinPilot Software v4.5, Applied Biosystem, USA) and nonlinear fitting methods to determine the false discovery rate (FDR) for peptide identification and quantification. Proteinpilot search parameters are shown in table $1[63,64]$. All reported proteins were based on the following conditions: (1) at least one peptide confidence $>95 \%$ (unused Prot Score $\geq 1.3$ ); (2) FDR $<1 \%$. The tobacco leaves samples with different maturity were compared between two (OM vs IM; OM vs WM; WM vs IM). (3) isolation interference for peptide spectrum matches (PSMs) $<40 \%$. The iTRAQ ratios and $p$-values were calculated using ProteinPilot. The software identified the median average protein ratio and corrected it to unity, and then applied this factor to all quantification results [65]. For protein quantification, it was necessary that a protein contained at least two unique peptides. The quantitative protein ratios were weighted and normalized by the median ratio in Mascot. In terms of missing value handling, the null values were maintained. Statistical analyses of data among groups were performed using Student's t-test. Proteins that had a ratio of $>1.2$ or $<0.83$ and $p$ -

Page $14 / 32$ 
values $<0.05$ were used as thresholds for determining differentially regulated proteins (DRPs) [66]. Additionally, the DRPs detected in only one material with at least two replicates were considered to be presence/absence regulated proteins. The biological coefficient of variation (CV) of each DRPs was also calculated.

Proteinpilot search parameters are shown in table 1区

\subsection{Bioinformatics Analysis}

Kyoto Encyclopedia of Genes and Genomes (http://www.genome.jp/kegg/) was used to annotate differentially expressed proteins to understand their biological functions. The KEGG enrichment pathway with Bonferroni-corrected $p$-value $<0.05$ was considered significant by using the hypergeometric test. All other pictures in this paper were drawn with R language (http://www.r-project.org/).

\subsection{PRM Quantitation Analysis}

Protein preparation was performed as described above. Targeted MS analysis using PRM (parallel reaction monitoring) was performed on a TripleTOF 5600 LC-MS/MS system (AB SCIEX, Massachusetts, USA). For PRM data collection, the list of optimized protein peptides is added to the inclusion list, and the mass spectrometer selects the size of the peptides in the list one by one to be broken and collects the secondary ion spectrum. All the peptide samples obtained by enzymatic hydrolysis were firstly mixed and then subjected to mass spectrometry DDA (Data Dependent Acquisition) detection. The Proteinpilot database was searched, and then the Skyline software was introduced to establish a spectrum library, and the peptides of the target protein were screened for method establishment. Design the PRM detection method and add the target peptide $\mathrm{m} / \mathrm{z}$ to the inclusion list to establish a mass spectrometry acquisition method. The PRM method is then used to perform PRM data acquisition on the mixed samples to adjust and optimize the PRM acquisition method to form a final PRM method for sample data collection. Each sample was dissolved with Loading Buffer ( $0.1 \%$ formic acid $/ 3 \%$ acetonitrile), and the supernatant was spotted after centrifugation. Data acquisition was performed on each sample using the optimized PRM mass spectrometry method described above to obtain 2 PRM spectral files. By extracting and analyzing these PRM spectrum files, quantitative information of the protein can be obtained. By extracting and analyzing these PRM spectrum files, quantitative information of the protein can be obtained $[67,68]$.

\section{Declarations}

\section{Ethics approval and consent to participate}

Not applicable.

\section{Consent for publication}


Not applicable.

\section{Availability of data and materials}

All data generated or analysed during this study are included in this published article [and its supplementary information files]

\section{Competing interests}

The authors declare that they have no competing interests. All authors approved the final manuscript.

\section{Funding}

This work was supported by the grant from Yunnan Provincial Tobacco Monopoly Bureau China (nos. 2019530000241019), the National Natural Science Foundation of China (Grant nos. 41601330), Yunnan Science and Technology Innovation Project (Grant nos.2019HB068), Yunnan Ten Thousand People Program (Congming Zou), Yunnan Applied Basic Research Projects (Grant nos.2017FB074). The founders did not play any roles in the design, analysis, and interpretation of this study or relevant data.

\section{Authors' Contributions}

$\mathrm{ZL}$ and $\mathrm{CZ}$ conceived the original research plans. $\mathrm{GZ}$ and $\mathrm{CH}$ designed the experiments. $\mathrm{PL}$ and $\mathrm{TX}$ performed the experiments. $\mathrm{YT}$ and $\mathrm{SS}$ analyzed the data. $\mathrm{YC}$ and $\mathrm{CH}$ wrote the manuscript. All authors reviewed and approved the final manuscript.

\section{Acknowledgments}

The authors wish to thank the Wuhan Gene Create Biological Engineering Co., Ltd who was of great help in the iTRAQ assays and data analysis.

\section{Abbreviations}

DEPs: differentially expressed proteins; iTRAQ: isobaric tag for relative and absolute quantification; PRM: parallel reaction monitoring; OM: over maturity; WM: well maturity; IM: immature; KEGG: Kyoto Encyclopedia of Genes and Genomes; DRPs: differentially regulated proteins;

\section{References}

1. Süssenbacher I, Menghini D, Scherzer G, Salinger K, Erhart T, Moser S, Vergeiner C, Hörtensteiner S, Kräutler B. Cryptic chlorophyll breakdown in non-senescent green Arabidopsis thaliana leaves. Photosynthesis Research. 2019; 142(1): 69-85.

2. Eyal T, Reinat N, Leah N, Smadar L, Vladimir K, Alon S, Yishai L, Yoram E, Ziv R, Zach A. Chlorophyll catabolism precedes changes in chloroplast structure and proteome during leaf senescence. Plant Direct. 2019; 3(3): e00127. 
3. Nam HG. The molecular genetic analysis of leaf senescence. Curr. Opin. Biotechnol. 1997; 8(2): 200207.

4. Nicole A, Bianka J, Klaus H. Epigenetic control of plant senescence and linked processes. Journal of Experimental Botany. 2014; 65(14): 3875-3887.

5. Yolcu S, Li XJ, Li SB, Kim YJ. Beyond the genetic code in leaf senescence. Journal of Experimental Botany, 2018, 69(4): 801-810.

6. Woo H R, Masclaux-Daubresse C, Lim P O. Plant senescence: how plants know when and how to die. 2018; 69(4): 715-718.

7. Chu MY, Yu YC. Research progress on factors affecting plant leaf senescence. Chinese Bulletin of Life Sciences. 2019; 31(02): 178-184.

8. Kim HJ, Hong SH, Kim YW, Lee IH, Jun JH, Phee B, Rupak T, Jeong H, Lee Y, Hong BS, Nam HG, Woo HR, Lim PO. Gene regulatory cascade of senescence-associated NAC transcription factors activated by ETHYLENE-INSENSITIVE2-mediated leaf senescence signalling in Arabidopsis. Journal of Experimental Botany. 2014; 65(14): 4023-4036.

9. Zhao JY, Li LL, Zhao YN, Zhao CX, Chen X, Liu PP, Zhou HN, Zhang JJ, Hu CX, Chen AG, Liu GS, Peng XJ, Lu X, Xu GW. Metabolic changes in primary, secondary, and lipid metabolism in tobacco leaf in response to topping. Analytical and Bioanalytical Chemistry. 2018; 410(3): 839-851.

10. Schöttler MA, Tóth SZ. Photosynthetic complex stoichiometry dynamics in higher plants: environmental acclimation and photosynthetic flux control. Front Plant Sci. 2014; 5: 188.

11. Sebastian FB, Alexander B, Philipp FWR, Holger S, Uwe C, Caspar JGJ. Extra-plastidial degradation of chlorophyll and photosystem I in tobacco leaves involving 'senescence-associated vacuoles'. The Plant Journal. 2019; 99(3): 465-477.

12. Nicole A, Bianka J, Klaus H. Epigenetic control of plant senescence and linked processes. Journal of Experimental Botany. 2014; 65(14):3875-3887.

13. Yolcu S, Li XJ, Li SB, Kim YJ. Beyond the genetic code in leaf senescence. Journal of Experimental Botany, 2018, 69(4): 801-810.

14. Lirui C, Xiaocui C, Cai HJ, Bing M, Min R, Ya ZC, Dan L, Rui MG, Ai GY. High-density SNP genetic linkage map construction and quantitative trait locus mapping for resistance to cucumber mosaic virus in tobacco (Nicotiana tabacum L.). The Crop Journal. 2019; 7(04): 539-547.

15. Zhao JY, Li LL, Zhao YN, Zhao CX, Chen X, Liu PP, Zhou HN, Zhang JJ, Hu CX, Chen AG, Liu GS, Peng XJ, Lu X, Xu GW. Metabolic changes in primary, secondary, and lipid metabolism in tobacco leaf in response to topping. Analytical and Bioanalytical Chemistry. 2018; 410(3): 839-851.

16. Jiang SP, Zhang ZY, Zheng SF, Liu DM, Li ZH, Ma YQ, Jiang MH. Effect of different harvesting maturity on tobacco quality with optimized leaf structure. Journal of Henan Agricultural Sciences. 2013; 42(11): 40-45.

17. Das PP, Lin Q, Wong SM. Comparative proteomics of Tobacco mosaic virus-infected Nicotiana tabacum plants identified major host proteins involved in photosystems and plant defence. Journal of proteomics. 2019; 194: 191-199. 
18. He X, Da HY, Heng Y, Ge B, Yi HZ, Bing GX. iTRAQ-based quantitative proteomic analysis reveals proteomic changes in leaves of cultivated tobacco (Nicotiana tabacum) in response to drought stress. Biochemical and Biophysical Research Communications. 2016; 469(3): 768-775.

19. Wang J, Wang XY, Zhou Q, Yang JM, Guo HX, Yang LJ, Liu WQ. iTRAQ protein profile analysis provides integrated insight into mechanisms of tolerance to TMV in tobacco (Nicotiana tabacum). Journal of Proteomics. 2016; 132: 21-30.

20. Altaf HM, Huygens F. Proteomic and bioinformatics tools to understand virulence mechanisms in Staphylococcus aureus. Current Proteomics. 2012; 9(1): 2-8.

21. Zhang H, Ge Y. Comprehensive analysis of protein modifications by top-down mass spectrometry. Circulation: Cardiovascular Genetics. 2011; 4(6): 711-711.

22. Judith $\mathrm{O}$, Jonas G, Peter G, Christophe D, Christophe L. iTRAQ-based analysis of changes in the cassava root proteome reveals pathways associated with post-harvest physiological deterioration. The Plant Journal. 2011; 67(1): 145-156.

23. Yang MM, Dong J, Zhao WC, Gao X. Characterization of proteins involved in early stage of wheat grain development by iTRAQ. Journal of Proteomics. 2016; 136: 157-166.

24. Elschenbroich $\mathrm{S}$, Kislinger T. Targeted proteomics by selected reaction monitoring mass spectrometry: applications to systems biology and biomarker discovery. Molecular Biosystems. 2011; 7(2): 292-303.

25. Bourmaud A, Gallien S, Domon B. Parallel reaction monitoring using quadrupole-Orbitrap mass spectrometer: principle and applications. Proteomics. 2016; 16(15-16): 2146-2159.

26. Elschenbroich $S$, and Kislinger $T$. Targeted proteomics by selected reaction monitoring mass spectrometry: applications to systems biology and biomarker discovery. Molecular BioSystems. 2011; 7: 292-303.

27. Bourmaud A, Gallien S, and Domon, B. Parallel reaction monitoring using quadrupole-orbitrap mass spectrometer: principle and applications. Proteomics. 2016; 16: 2146-2159.

28. Pino LK, Searle BC, Bollinger JG, Nunn B, MacLean B, MacCoss MJ. The Skyline ecosystem: Informatics for quantitative mass spectrometry proteomics. Mass Spectrometry Reviews. 2017.

29. Yi JH. Determination and Study on Photosynthetic Parameters of Euonymus japonicus. Technology Wind. 2018; 201-202.

30. Canales FJ, De la Haba P, Barrientos E, Agüera E. Effect of CO2 enrichment and increased nitrogen supply on the induction of sunflower (Helianthus annuus L.) primary leaves senescence. Canadian Journal of Plant Science. 2016; 96(6): 1002-1013.

31. Mayta ML, Lodeyro AF, Guiamet JJ, Tognetti VB, Melzer M, Hajirezaei MR, Carrillo N. Expression of a plastid-targeted flavodoxin decreases chloroplast reactive oxygen species accumulation and delays senescence in aging tobacco leaves. Frontiers in plant science. 2018; 9: 1039.

32. Kim J. Sugar metabolism as input signals and fuel for leaves senescence. Genes \& Genomics. 2019; 41(7): 737-746. 
33. Wingler A, Roitsch T. Metabolic regulation of leaves senescence: interactions of sugar signalling with biotic and abiotic stress responses. Plant Biology. 2008; 10: 50-62.

34. Zhang AH, Lu QT, Yin Y, Ding SH, Wen XG, Lu CM. Comparative proteomic analysis provides new insights into the regulation of carbon metabolism during leaves senescence of rice grown under field conditions. Journal of Plant Physiology. 2010; 167(16): 1380-1389.

35. Ma GY, Shi XH, Zou QC, Tian DQ, An X, Zhu KY. iTRAQ-based quantitative proteomic analysis reveals dynamic changes during daylily flower senescence. Planta. 2018; 248: 859-873.

36. McKinley BA, Casto AL, Rooney WL, Mullet JE. Developmental dynamics of stem starch accumulation in Sorghum bicolor. Plant Direct. 2018; 2(8): e00074.

37. Van Doorn WG. Is the onset of senescence in leaves cells of intact plants due to low or high sugar levels? Journal of Experimental Botany. 2008; 59(8): 1963-1972.

38. Hasnahana C, Debajyoti K, Biju B, Sunita O, Manash PB, Dharitri S, Tinka S, Ponnala VM, Pragya S, Utpal B. Exploring the Benefits of Endophytic Fungi via Omics[M]//Advances in Endophytic Fungal Research. Springer, Cham. 2019: 51-81.

39. Gustavo S, Gabriel M, Ma. del CO, Bernard RG. Plant growth-promoting bacterial endophytes. Microbiological Research. 2016; 183: 92-99.

40. Li WJ, Qian ZQ, Jin R, Xu FH, Zhu K,Yang MZ, Chen SY. Microbiology China. 2013; 40(05): 783-791.

41. Zhang L, Wang Z, Zhang YJ, Lin C, Chen YP, Li JY, Mao ZC. Proteomic Analysis of Senescing Leaves of Tobacco. Plant Physiology Journal. 2014; 50(04): 488-500.

42. Martin C, Thimann KV. The role of protein synthesis in the senescence of leaves: I. The formation of protease. Plant physiology. 1972; 49(1): 64-71.

43. Amunts A, Toporik H, Borovikova A, Nelson N. Structure determination and improved model of plant photosystem I. Journal of Biological Chemistry. 2010; 285(5): 3478-3486.

44. Rochaix JD. Role of thylakoid protein kinases in photosynthetic acclimation. Febs Letters 2007; 581(15): 2768-2775.

45. Nishiyama Y, Allakhverdiev SI., Murata N. A new paradigm for the action of reactive oxygen species in the photoinhibition of photosystem II. Biochimica et Biophysica Acta (BBA)-Bioenergetics. 2006; 1757(7): 742-749.

46. Plaxton WC. The organization and regulation of plant glycolysis. Annual Review of Plant Biology.1996; 47: 185-214.

47. Joshi R, Karan R, Singla-Pareek SL, Pareek A. Ectopic expression of Pokkali phosphoglycerate kinase-2 (OsPGK2-P) improves yield in tobacco plants under salinity stress. Plant Cell Reports. 2016; 35(1): 27-41.

48. Nelson N, Junge W. Structure and energy transfer in photosystems of oxygenic photosynthesis. Annual Review of Biochemistry. 2015; 84: 659-683.

49. Mi HL. The regulation of NAD (P)H dehydrogenase complexes bound in thylakoid membranes in photosynthesis. Plant Physiology Journal. 2016; 52(10):1457-1465. 
50. Xu Y, Gianfagna T, Huang B. Proteomic changes associated with expression of a gene (ipt) controlling cytokinin synthesis for improving heat tolerance in a perennial grass species. Journal of Experimental Botany. 2010; 61(12): 3273-3289.

51. Sorek M, Yacobi Y Z, Roopin M, Berman-Frank I, Levy O. Photosynthetic circadian rhythmicity patterns of Symbiodium, the coral endosymbiotic algae. Proceedings of the Royal Society B: Biological Sciences. 2013; 280(1759): 20122942.

52. Yamada K, Lim J, Dale JM, Chen HM, Shinn P, Palm CJ, Southwick AM, Wu HC, Kim C, Nguyen M, Pham P, Cheuk R, Karlin-Newmann G, Liu SX, Lam B, Sakano H, Wu T, Yu G, Miranda M, Quach HL, Tripp M, Chang CH, Lee JM, Toriumi M, Chan MMH, Tang CC, Onodera CS, Deng JM, Akiyama K, Ansari Y, Arakawa T, Banh J, Banno F, Bowser L, Brooks SC. Empirical analysis of transcriptional activity in the Arabidopsis genome. Science. 2003; 302(5646): 842-846.

53. Sugihara K, Hanagata N, Dubinsky Z, Baba S, Karube I. Molecular characterization of cDNA encoding oxygen evolving enhancer protein 1 increased by salt treatment in the mangrove Bruguiera gymnorrhiza. Plant and Cell Physiology. 2000; 41(11):1279-185.

54. Fatehi F, Hosseinzadeh A, Alizadeh H, Brimavandi T. The proteome response of Hordeum spontaneum to salinity stress. Cereal Research Communications. 2012; 41(1): 78-87.

55. Ngamhui N, Akkasaeng C, ZhuYJ, Tantisuwichwong N, Roytrakul S, Sansayawichai T. Differentially expressed proteins in sugarcane leaves in response to water deficit stress. Plant Omics. 2012; 5(4): 365-371.

56. Wassim A, Ichrak BR, Saïda A. Putative role of proteins involved in detoxification of reactive oxygen species in the early response to gravitropic stimulation of poplar stems. Plant Signaling \& Behavior. 2013; 8(1): e22411.

57. Girard-Bascoul J, Choquet Y, Schneider M, Delosmel M, Dron M. Characterization of a chloroplast mutation in the psaA2 gene of Chlamydomonas reinhardtii. Current Genetics. 1987; 12(7): 489-495.

58. Lefebvre-Legendre L, Choquet $Y$, Kuras R, Loubéry S, Douchi D, Goldschmidt-Clermont M. A nucleusencoded chloroplast protein regulated by iron availability governs expression of the photosystem I subunit PsaA in Chlamydomonas reinhardtii. Plant Physiology. 2015; 167(4): 1527-1540.

59. Teramoto H, Nakamori A, Minagawa J, Ono T. A. Light-intensity-dependent expression of Lhc gene family encoding light-harvesting chlorophyll-a/b proteins of photosystem II in Chlamydomonas reinhardtii. Plant Physiology. 2002; 130(1): 325-333.

60. Qin HJ. Effects of Photosynthetic Characteristics of Three Elymus Forage Grasses under Drought Stress. Grassland and Prataculture. 2019; 31(02): 48-52.

61. Li CL, Xu HM, Xu J, Chun XX, Ni DJ. Effects of aluminium on ultrastructure and antioxidant activity in leaves of tea plant. Acta physiologiae plantarum. 2011; 33(3): 973-978.

62. Kruger NJ. The Bradford Method For Protein Quantitation. Methods in Molecular Biology (Clifton, N.J.). 1994; 32:9-15.

63. Liu PF, Du YS, Meng LJ, Li X, Liu Y. Proteomic analysis in kidneys of Atlantic salmon infected with Aeromonas salmonicida by iTRAQ. Developmental \& Comparative Immunology, 2017, 72: 140-153. 
64. Zhang C, Zhu Y, Liu YG, Zhang XG, Yue QN, Li L, Chen YT, Lu S, Teng ZW. SEMA3B-AS1-inhibited osteogenic differentiation of human mesenchymal stem cells revealed by quantitative proteomics analysis. Journal of Cellular Physiology, 2019, 234(3): 2491-2499.

65. Wang XS, Jones DR, Shaw TI, Cho J, Wang YY, Tan HY, Xie B, Zhou SP, Li YX, Peng JM. Target-decoybased false discovery rate estimation for large-scale metabolite identification. Journal of Proteome Research.2018; 17: 2328-2334.

66. Tang WH, Shilov IV, Seymour SL. Nonlinear ftting method for determining local false discovery rates from decoy database searches, Journal of Proteome Research. 2008; 7(9): 3661-3667.

67. Wu LC, Wang SX, Tian L, Wu LJ, Li MN, Zhang J, Li P, Zhang WQ, Chen YH. Comparative proteomic analysis of the maize responses to early leaf senescence induced by preventing pollination. Journal of Proteomics. 2018; 177: 75-87

68. Tang XY, Wang HM, Long QT, Huang YL, Lu M, Cao ZB, Wang JL. Identification of Cold Responsive Proteins in Dongxiang Wild Rice Root Based on iTRAQ Technique. Molecular Plant Breeding. 2019; 17(11): 3624-3631.

69. Luczak M, Formanowicz D, Marczak Ł, Suszyńska-Zajczyk J, Pawliczak E, Wanic-Kossowska M, Stobiecki M. iTRAQ-based proteomic analysis of plasma reveals abnormalities in lipid metabolism proteins in chronic kidney disease-related atherosclerosis. Scientific Reports, 2016, 6: 32511.

70. Vidova V, Spacil Z. A review on mass spectrometry-based quantitative proteomics: Targeted and data independent acquisition. Analytica Chimica Acta. 2017; 964: 7-23.

\section{Table}

Table 1 Proteinpilot searching parameters

Item

Type of search

Enzyme

Cys Alkylation

Instrument

Bias Correction

Background Correction
Value

iTRAQ 8plex (Peptide Labeled )

Trypsin

Iodoacetamide

TripleTOF 5600

TRUE

TRUE
ID focus

Search Effort

Protein Mass

Database
Biological modifications

Thorough ID

Unrestricted uniprot-taxonomy\%3A-Nicotiana+tabacum.fasta $[0$ sequence in total $\square$

\section{Additional File Legends}


Additional file 1: Table S1. Pathway enrichment of differentially expressed proteins between OM and IM

Additional file 2: Table S2. Pathway enrichment of differentially expressed proteins between OM and WM

Additional file 3: Table S3. Pathway enrichment of differentially expressed proteins between WM and IM

\section{Figures}

A

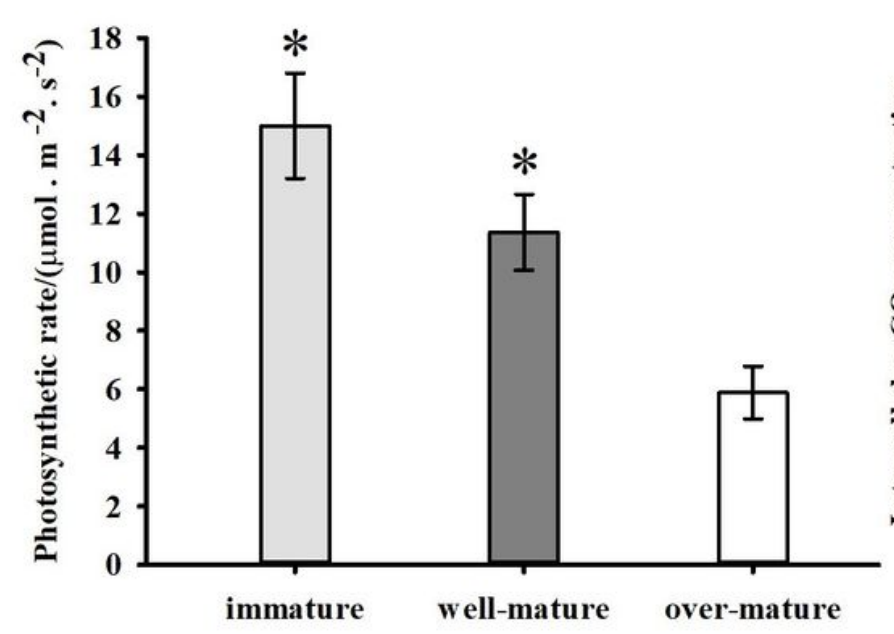

$\mathrm{C}$

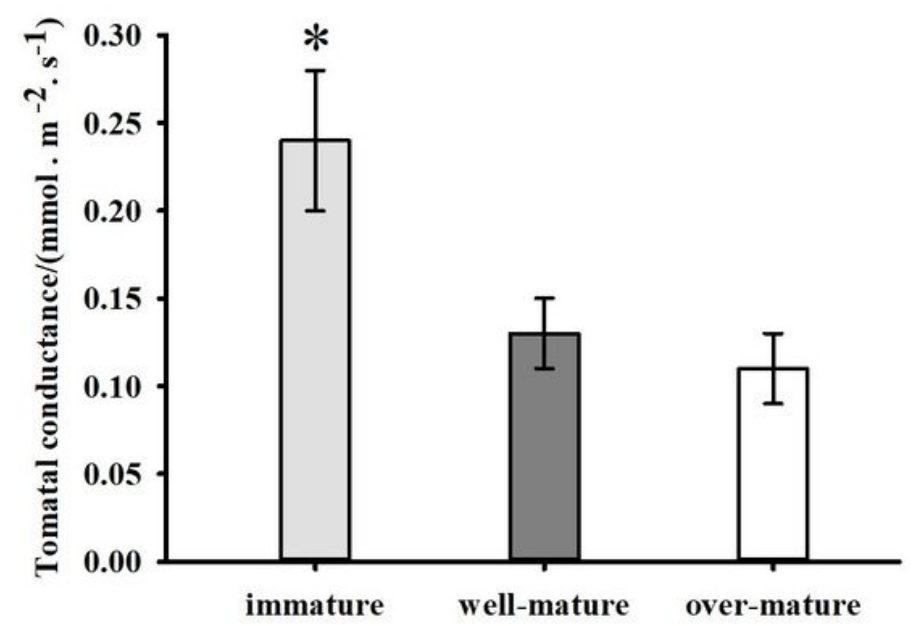

B

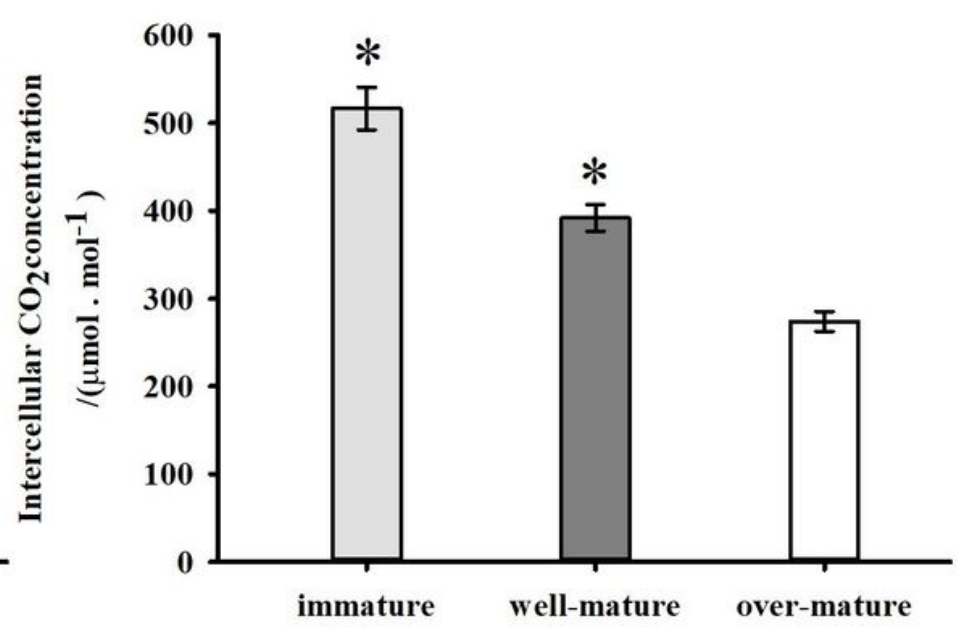

$\mathrm{D}$

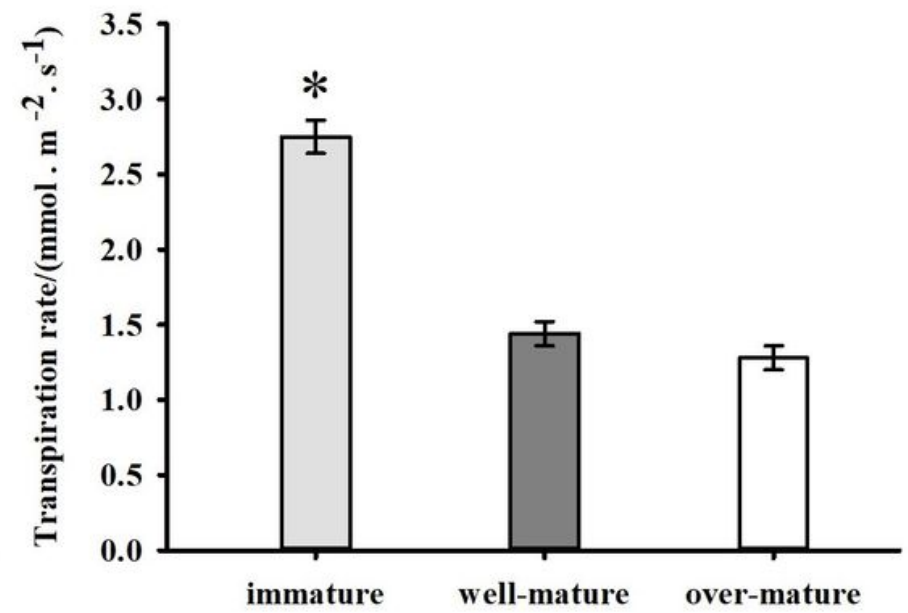

Figure 1

Photosynthesis at different maturity stages Note: (A) Photosynthetic rate; (B) Intercellular CO2 concentration; (C) Stomatal conductance; (D) Transpiration rate; ${ }^{*}$ ) represent significant differences at $p<0.05$ level between the groups, and error bars show the standard error of the means $(n=3)$ 

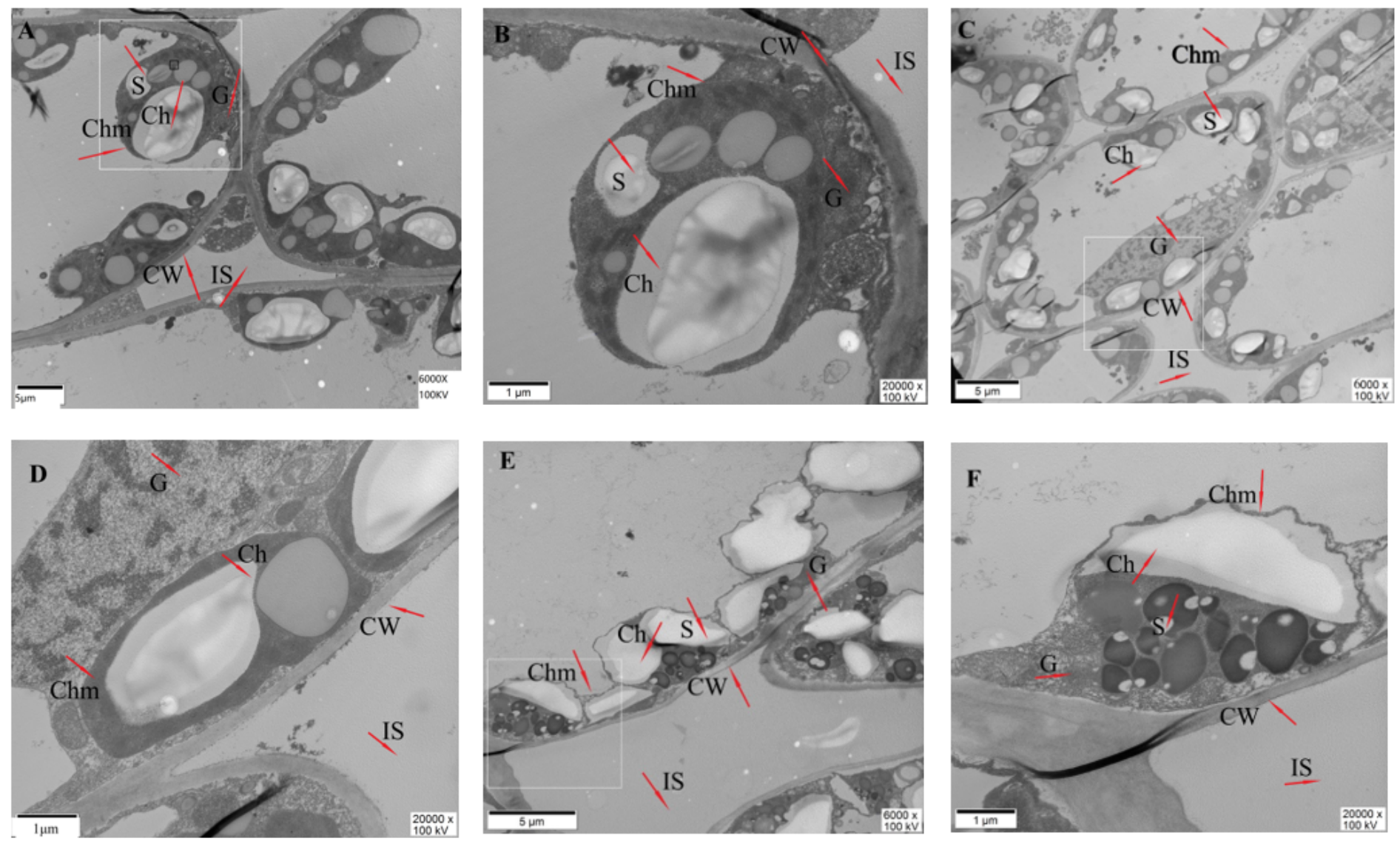

\section{Figure 2}

Mesophyll cell and chloroplast ultrastructure changes in tobacco leaves Note: The left column (A, C, E) shows the chlorophyll mesophyll cells and topography $(\times 6000)$. Shown in the right column $(B, D, F)$ is the ultrastructure $(\times 20,000)$ of mesophyll cells and chloroplast. Micrographs in rows $A-B, C-D$, and $E-F$, respectively, were from plants IM, WM and OM. IS, Intercellular space; CW, cell wall; Ch, Chloroplast; $\mathrm{S}$, starch granule; Chm, chloroplast membrane; G, Grana lamellae. 

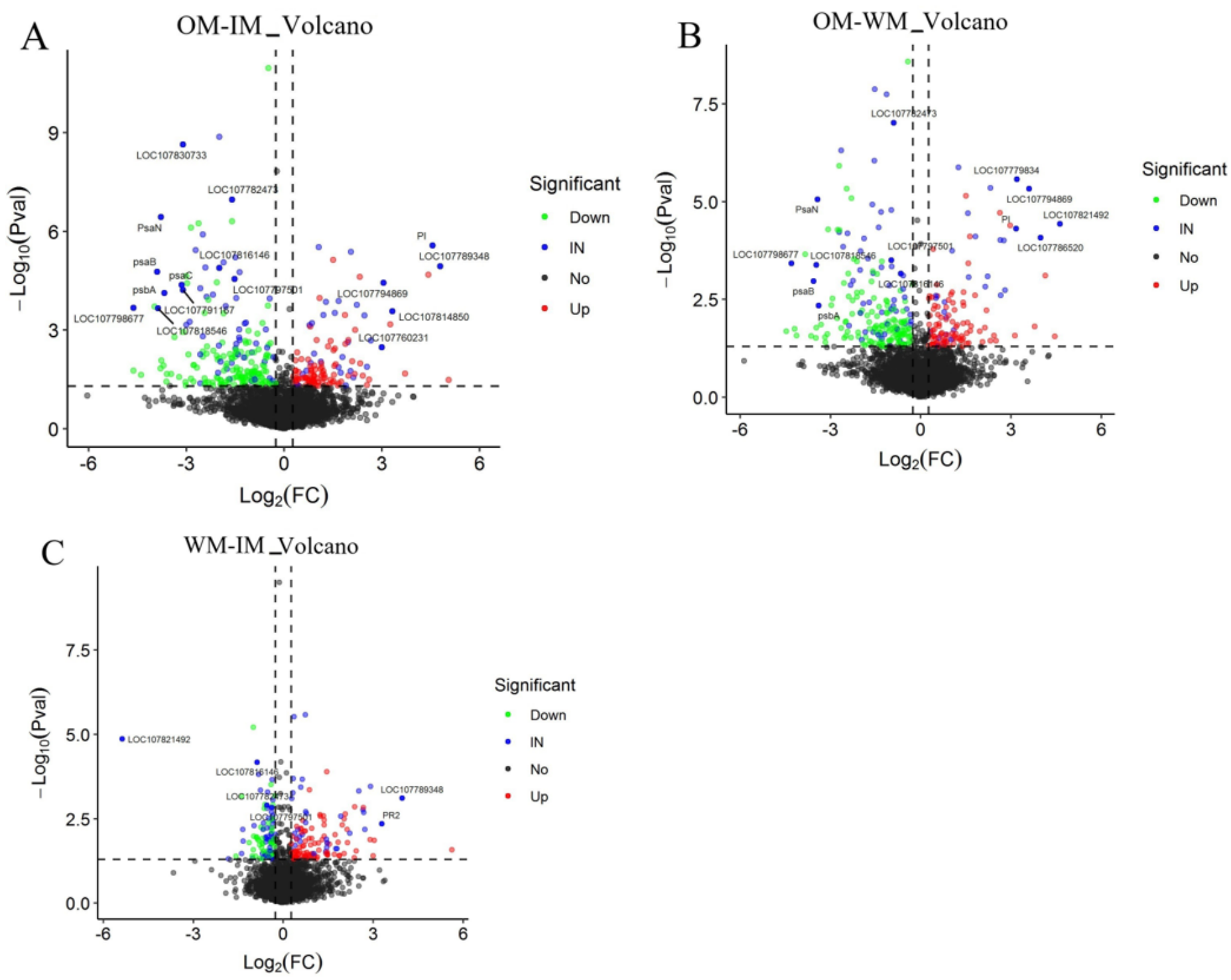

Figure 3

The volcano plot shows the collected p-values for the changes in the patterns of all identified proteins in pairwise comparisons Note: (A) DEPs in OM vs IM; (B) DEPs in OM vs WM; (C) DEPs in WM vs IM. Black spots are unchanged proteins, Green spots represent down-regulated proteins and the red spots represent up-regulated protein. Blue spots represent candidates with a putative role. 


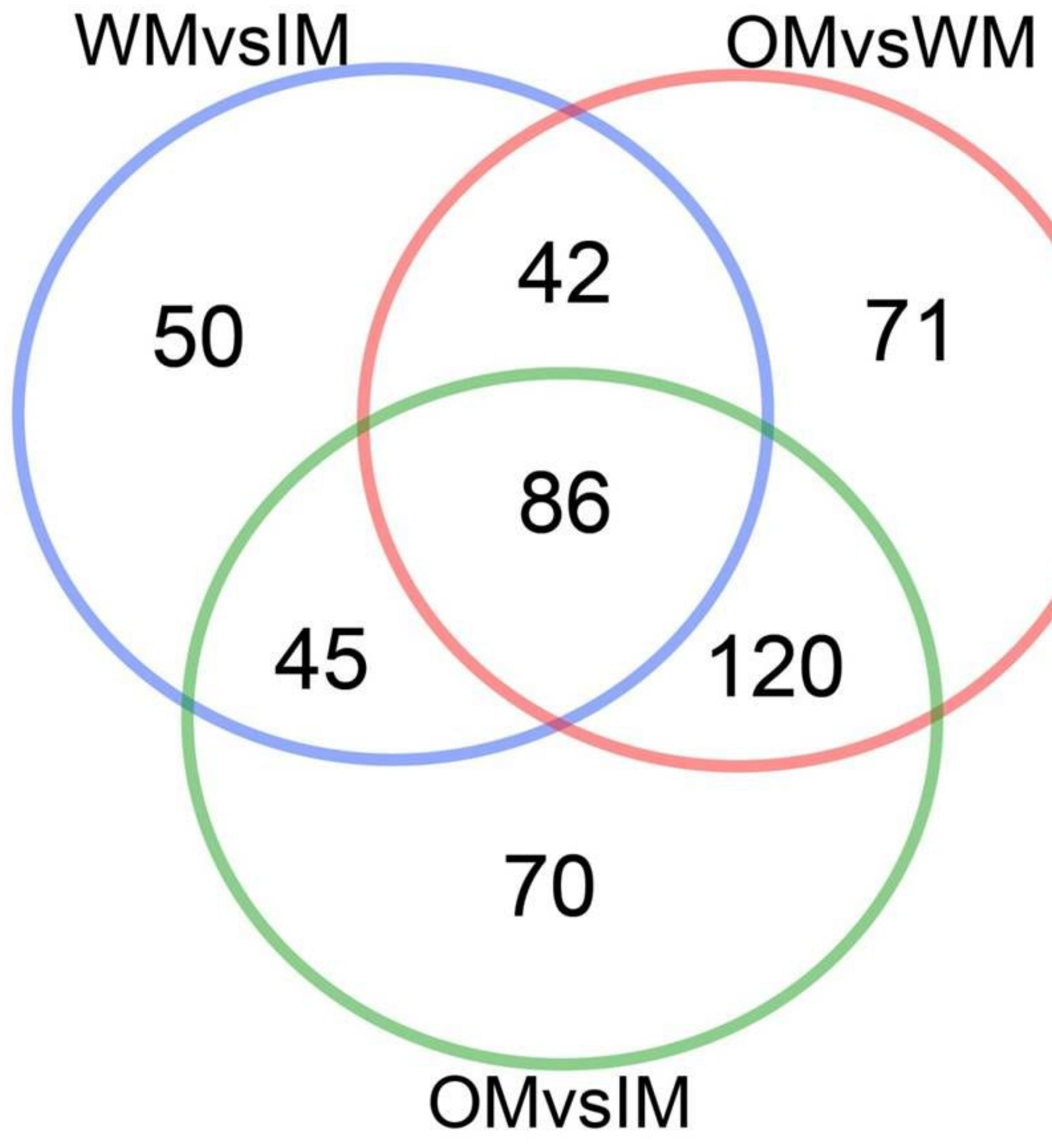

Figure 4

Venn diagram of differentially expressed proteins between different groups 
A
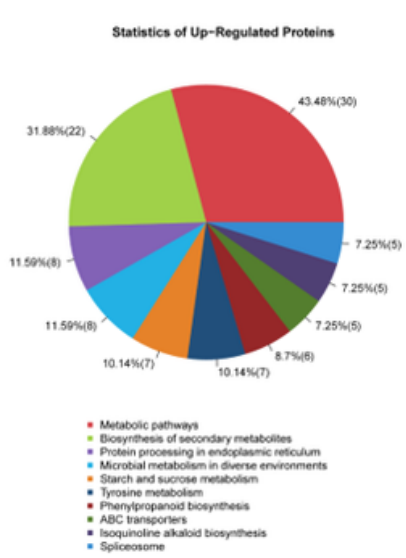

C
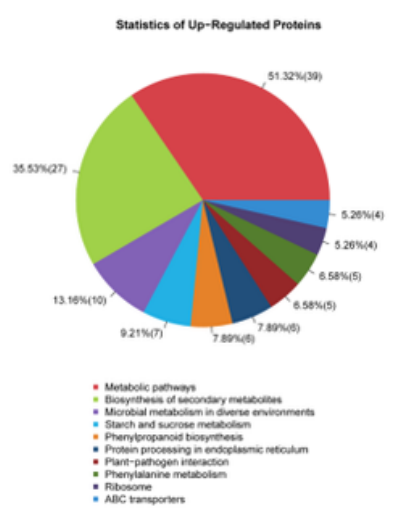

B
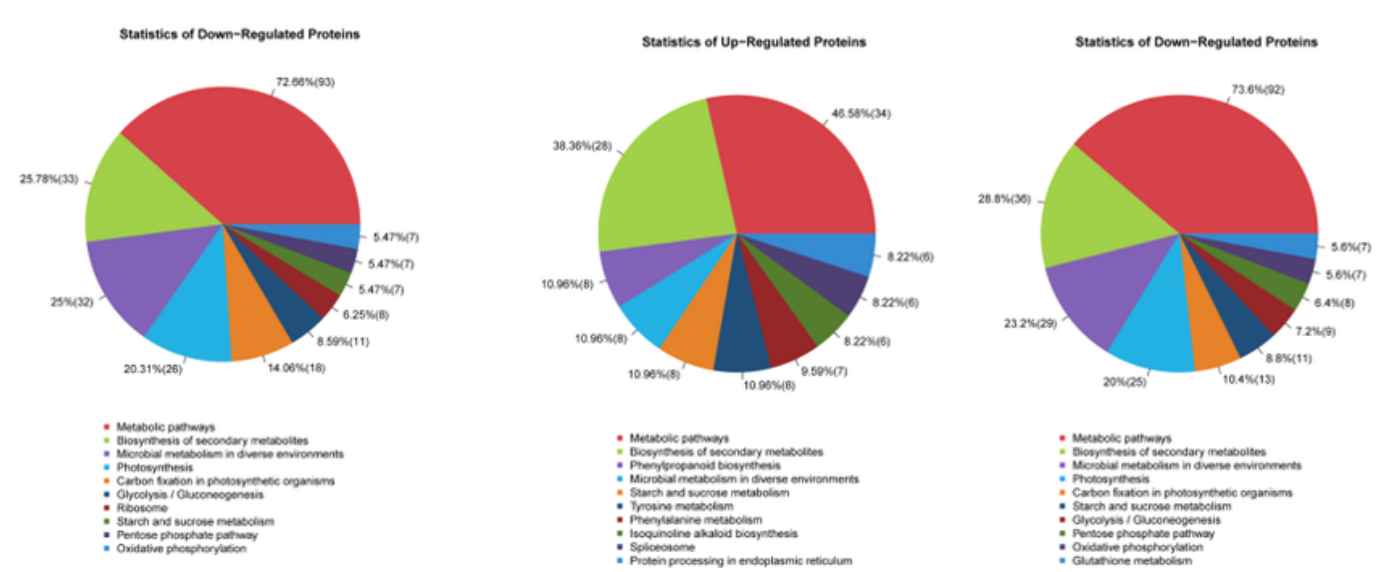
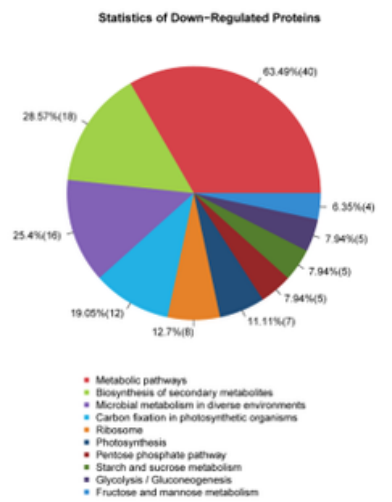

\section{Figure 5}

The top ten metabolic pathways involved by three groups of differential proteins Note: (A) OM vs IM metabolic pathways involved by differential proteins. (B) OM vs WM metabolic pathways involved by differential proteins. (C) WM vs IM metabolic pathways involved by differential proteins. 


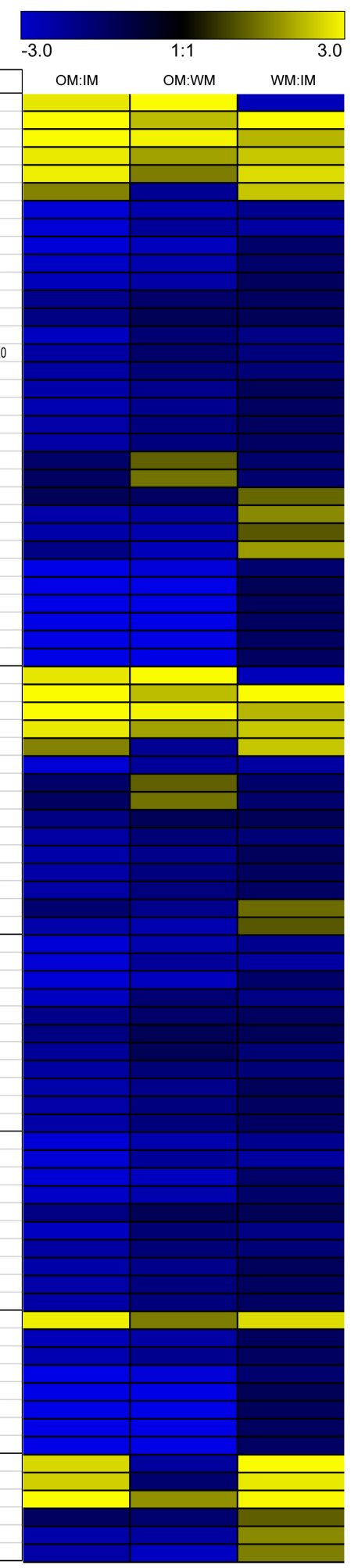

\begin{tabular}{|c|c|c|c|c|c|c|}
\hline Pathway & Protein_id & Gnen_id & Decriptions & OM:IM & OM:WM & WM:IM \\
\hline \multirow{32}{*}{ Metabolic pathways } & A0A1S3ZGA9 & LOC107786520 & polyphenol oxidase E, chloroplastic-like & & & \\
\hline & AOA1S3ZQP6 & LOC107789348 & polyphenol oxidase $\mathrm{E}$, chloroplastic-like isoform $\mathrm{X} 1$ & & & \\
\hline & A0A1S3X216 & LOC107760231 & polyphenol oxidase E, chloroplastic-like & & & \\
\hline & AOA1S3YJ62 & LOC107776586 & acetylajmalan esterase-like & & & \\
\hline & AOA1S4DCM1 & LOC107828191 & oxygen-evolving enhancer protein 3-2, chloroplastic-like & & & \\
\hline & A0A1S4ABD1 & LOC107795792 & farnesyl pyrophosphate synthase 1-like & & & \\
\hline & A0A1S3ZY67 & LOC107791770 & Ribulose bisphosphate carboxylase small chain & & & \\
\hline & AOA1S3Z8U8 & LOC107784214 & Fructose-bisphosphate aldolase & & & \\
\hline & Q84QE5 & LOC107771723 & Ribulose bisphosphate carboxylase small chain & & & \\
\hline & AOA1S4CNKO & LOC107820790 & Glyceraldehyde-3-phosphate dehydrogenase & & & \\
\hline & AOA140G1P8 & atpF & ATP synthase CFO B subunit & & & \\
\hline & AOA1S4BWK5 & LOC107812643 & NADP-dependent glyceraldehyde-3-phosphate dehydrogenase-like & & & \\
\hline & AOA1S4AGC9 & LOC107797391 & transketolase, chloroplastic & & & \\
\hline & AOA1S4C882 & LOC107816146 & sedoheptulose-1,7-bisphosphatase, chloroplastic-like & & & \\
\hline & AOA1S3X3J1 & LOC107760931 & haloacid dehalogenase-like hydrolase domain-containing protein At3g48420 & & & \\
\hline & AOA1S3XXM3 & LOC107769721 & triosephosphate isomerase, chloroplastic-like & & & \\
\hline & AOA1S4AGWO & LOC107797501 & malate dehydrogenase, glyoxysomal & & & \\
\hline & AOA140G1S1 & atpE & ATP synthase CF1 epsilon subunit & & & \\
\hline & F2VJ75 & LOC107797422 & Fructose-bisphosphate aldolase & & & \\
\hline & AOA1S4DNI7 & LOC107831470 & ribulose-phosphate 3-epimerase, chloroplastic & & & \\
\hline & Q0MW94 & DXR & 1-deoxy-D-xylulose-5-phosphate reductoisomerase & & & \\
\hline & A0A1S3Y911 & LOC107773751 & protein tas-like & & & \\
\hline & AOA1S4ANB9 & LOC107799508 & peroxisomal (S)-2-hydroxy-acid oxidase-like & & & \\
\hline & A0A1S3Z2T8 & LOC107782372 & Pectinesterase & & & \\
\hline & J7G1TO & VDE & Violaxanthin de-epoxidase & & & \\
\hline & AOA1S4DFE6 & LOC107829039 & Lipoxygenase & & & \\
\hline & AOA140G1X0 & $\mathrm{psaC}$ & Photosystem l iron-sulfur center & & & \\
\hline & A0A140G1P2 & $\mathrm{psbA}$ & Photosystem II protein D1 & & & \\
\hline & D2K7Z2 & Psan & Photosystem I reaction center subunit & & & \\
\hline & AOA140G1R2 & psaB & Photosystem I P700 chlorophyll a apoprotein A2 & & & \\
\hline & AOA1S4CFV4 & LOC107818546 & photosystem I reaction center subunit IV A, chloroplastic & & & \\
\hline & AOA1S4AKK6 & LOC107798677 & Chlorophyll a-b binding protein, chloroplastic & & & \\
\hline \multirow{15}{*}{$\begin{array}{l}\text { Biosynthesis of secondary } \\
\text { metabolites }\end{array}$} & A0A1S3ZGA9 & LOC107786520 & polyphenol oxidase E, chloroplastic-like & & & \\
\hline & AOA1S3ZQP6 & LOC107789348 & polyphenol oxidase $\mathrm{E}$, chloroplastic-like isoform X1 & & & \\
\hline & A0A1S3X216 & LOC107760231 & polyphenol oxidase E, chloroplastic-like & & & \\
\hline & A0A1S3YJ62 & LOC107776586 & acetylajmalan esterase-like & & & \\
\hline & AOA1S4ABD1 & LOC107795792 & farnesyl pyrophosphate synthase 1-like & & & \\
\hline & A0A1S3Z8U8 & LOC107784214 & Fructose-bisphosphate aldolase & & & \\
\hline & Q0MW94 & DXR & 1-deoxy-D-xylulose-5-phosphate reductoisomerase & & & \\
\hline & AOA1S3Y9|1 & LOC107773751 & protein tas-like & & & \\
\hline & AOA1S4AGC9 & LOC107797391 & transketolase, chloroplastic & & & \\
\hline & A0A1S3XXM3 & LOC107769721 & triosephosphate isomerase, chloroplastic-like & & & \\
\hline & AOA1S4AGWO & LOC107797501 & malate dehydrogenase, glyoxysomal & & & \\
\hline & F2VJ75 & LOC107797422 & Fructose-bisphosphate aldolase & & & \\
\hline & A0A1S4DNI7 & LOC107831470 & ribulose-phosphate 3-epimerase, chloroplastic & & & \\
\hline & AOA1S3Z7D3 & LOC107783636 & 7-deoxyloganetic acid glucosyltransferase-like & & & \\
\hline & J7G1T0 & VDE & Violaxanthin de-epoxidase & & & \\
\hline \multirow{11}{*}{$\begin{array}{l}\text { Microbial metabolism in } \\
\text { diverse environments }\end{array}$} & A0A1S3ZY67 & LOC107791770 & Ribulose bisphosphate carboxylase small chain & & & \\
\hline & A0A1S3Z8U8 & LOC107784214 & Fructose-bisphosphate aldolase & & & \\
\hline & Q84QE5 & LOC107771723 & Ribulose bisphosphate carboxylase small chain & & & \\
\hline & AOA1S4C882 & LOC107816146 & sedoheptulose-1,7-bisphosphatase, chloroplastic-like & & & \\
\hline & A0A1S4BWK5 & LOC107812643 & NADP-dependent glyceraldehyde-3-phosphate dehydrogenase-like & & & \\
\hline & A0A1S4AGC9 & LOC107797391 & transketolase, chloroplastic & & & \\
\hline & A0A1S3X073 & LOC107759700 & phosphoglycolate phosphatase $1 \mathrm{~B}$, chloroplastic-like & & & \\
\hline & A0A1S3XXM3 & LOC107769721 & triosephosphate isomerase, chloroplastic-like & & & \\
\hline & AOA1S4AGWO & LOC107797501 & malate dehydrogenase, glyoxysomal & & & \\
\hline & F2VJ75 & LOC107797422 & Fructose-bisphosphate aldolase & & & \\
\hline & A0A1S4DNI7 & LOC107831470 & ribulose-phosphate 3-epimerase, chloroplastic & & & \\
\hline \multirow{10}{*}{$\begin{array}{c}\text { Carbon fixation in } \\
\text { photosynthetic organisms }\end{array}$} & A0A1S3ZY67 & LOC107791770 & Ribulose bisphosphate carboxylase small chain & & & \\
\hline & A0A1S3Z8U8 & LOC107784214 & Fructose-bisphosphate aldolase & & & \\
\hline & Q84QE5 & LOC107771723 & Ribulose bisphosphate carboxylase small chain & & & \\
\hline & AOA1S4CNKO & LOC107820790 & Glyceraldehyde-3-phosphate dehydrogenase & & & \\
\hline & AOA1S4AGC9 & LOC107797391 & transketolase, chloroplastic & & & \\
\hline & AOA1S4C882 & LOC107816146 & sedoheptulose-1,7-bisphosphatase, chloroplastic-like & & & \\
\hline & AOA1S3XXM3 & LOC107769721 & triosephosphate isomerase, chloroplastic-like & & & \\
\hline & AOA1S4AGWO & LOC107797501 & malate dehydrogenase, glyoxysomal & & & \\
\hline & F2VJ75 & LOC107797422 & Fructose-bisphosphate aldolase & & & \\
\hline & AOA1S4DNI7 & LOC107831470 & ribulose-phosphate 3-epimerase, chloroplastic & & & \\
\hline & A0A1S4DCM1 & LOC107828191 & oxygen-evolving enhancer protein 3-2, chloroplastic-like & & & \\
\hline & A0A140G1P8 & atpF & ATP synthase CFO B subunit & & & \\
\hline & A0A140G1S1 & atpE & ATP synthase CF1 epsilon subunit & & & \\
\hline & A0A140G1X0 & psac & Photosystem I iron-sulfur center & & & \\
\hline Photosynthesis & AOA140G1P2 & $\mathrm{psbA}$ & Photosystem II protein D1 & & & \\
\hline & D2K7Z2 & Psan & Photosystem I reaction center subunit & & & \\
\hline & A0A140G1R2 & psaB & Photosystem I P700 chlorophyll a apoprotein A2 & & & \\
\hline & AOA1S4CFV4 & LOC107818546 & photosystem I reaction center subunit IV A, chloroplastic & & & \\
\hline & P23547 & PR2 & Glucan endo-1,3-beta-glucosidase, acidic isoform G19 & & & \\
\hline & AOA1S3XHXB & LOC107765187 & glucan endo-1,3-beta-glucosidase, acidic-like & & & \\
\hline Starch and sucrose & A0A1S4C433 & LOC107814850 & glucan endo-1,3-beta-glucosidase, acidic & & & \\
\hline metabolism & AOA1S4C3L2 & LOC107814807 & Alpha-1,4 glucan phosphorylase & & & \\
\hline & AOA1S322T8 & LOC107782372 & Pectinesterase & & & \\
\hline & A0A1S4AT57 & LOC107801151 & glucan endo-1,3-beta-glucosidase, basic vacuolar & & & \\
\hline
\end{tabular}

\section{Figure 6}

Differential protein relative expression matrix heatmap of the three groups Note: The color in the figure indicates the relative expression level of the protein in the sample. The yellow color indicates that the protein has a higher expression level in the sample, and the blue color represents a lower expression level. Color bars represent specific expression abundance. 

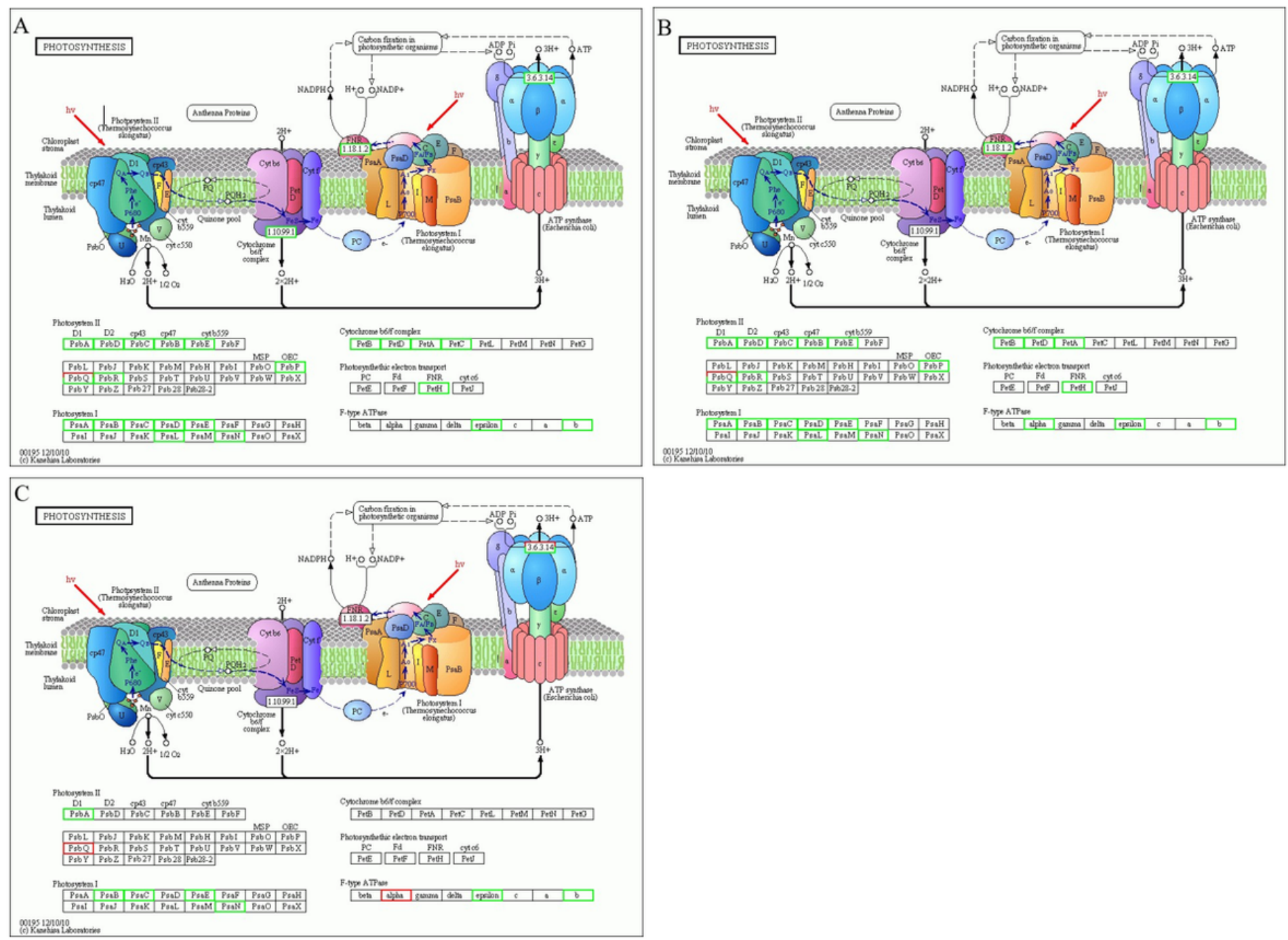

\section{Figure 7}

Representative significantly enriched photosynthesis related KEGG pathways Note: (A) The OM vs IM significantly enriched photosynthesis pathway; (B) the OM vs WM significantly enriched photosynthesis pathway; (C) the WM vs IM significantly enriched photosynthesis pathway. The abundance of proteins is represented by different colors, with red for up-regulated, green for down-regulated, and black for stable expression of proteins. 

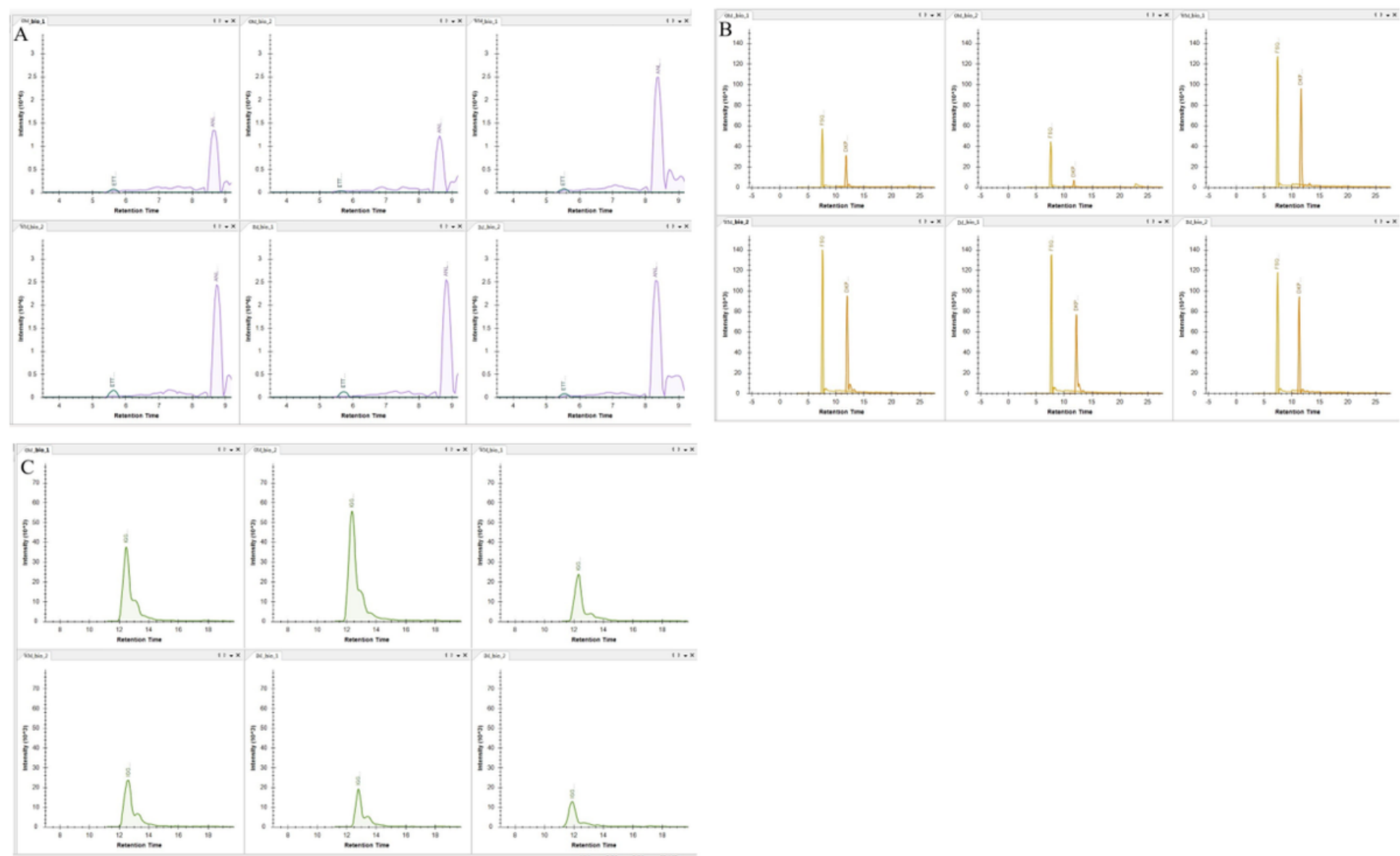

Figure 8

Visualization of skyline peptide results Note: (A) The ratios of A0A140G1P2; (B) the ratios of AOA140G1R2; (C) the ratios of A0A1S4DCM1. 


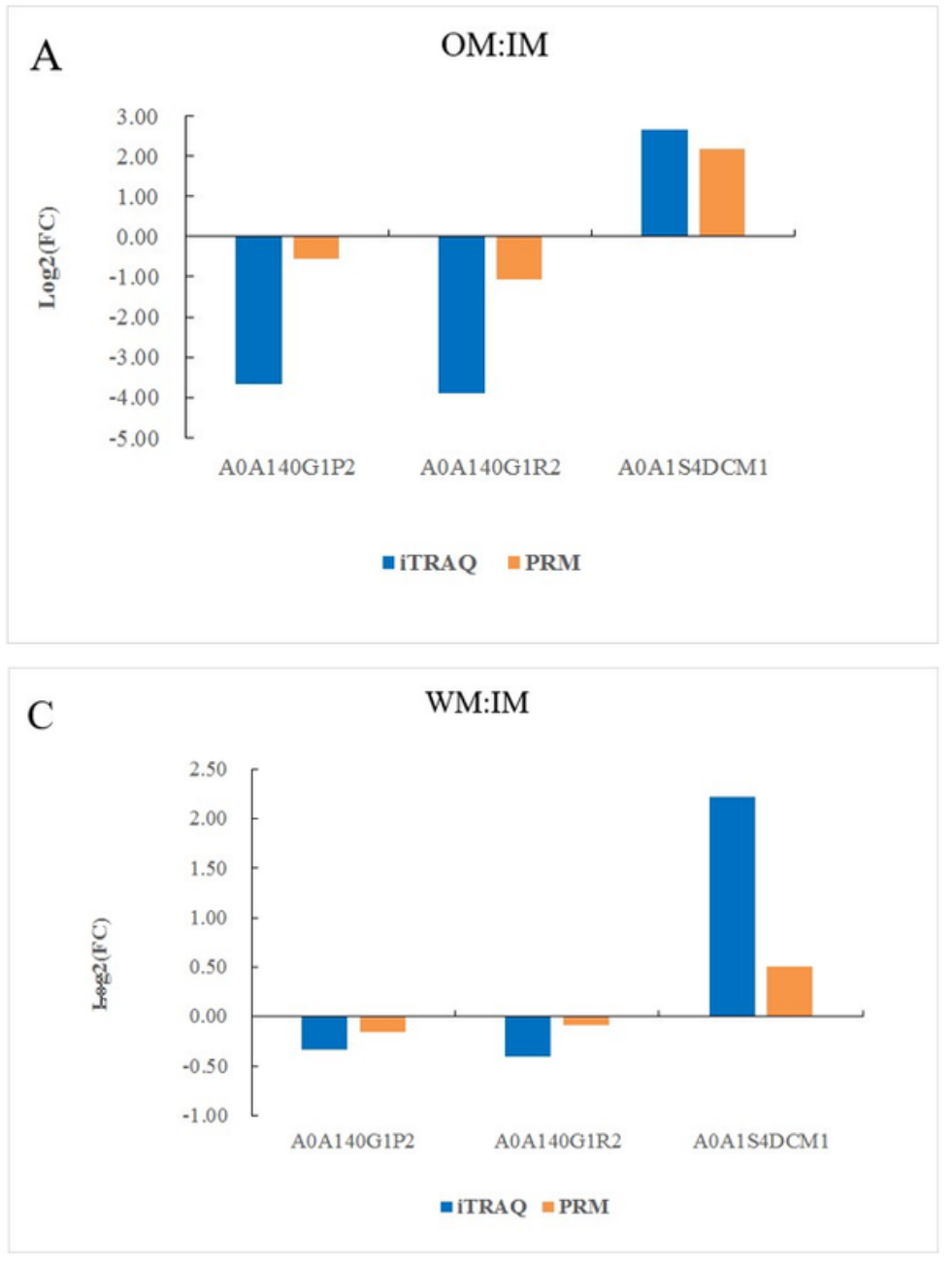

$\mathrm{B}$

OM:WM

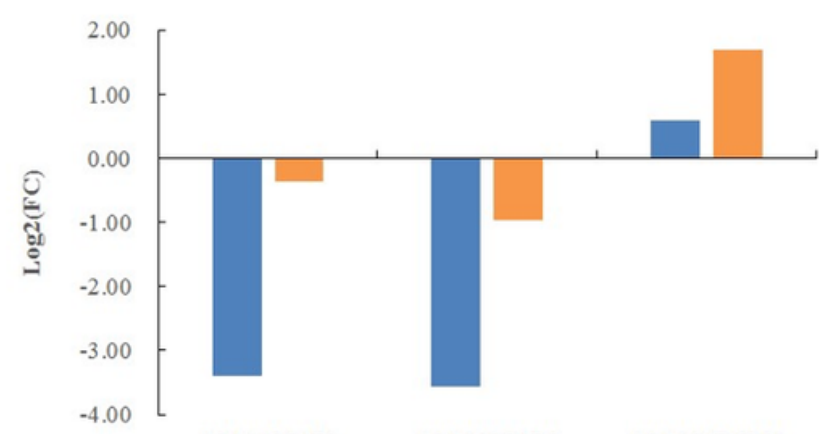

A0A140G1P2 A0A140G1R2 A0A1S4DCM1

miTRAQ $=$ PRM

\section{Figure 9}

Column map of PRM quantitative protein differences among different samples Note: (A) OM vs IM; (B) OM vs WM; (C) WM vs IM. The proteins corresponding to the column on the upside of transverse axis 0 represent up-regulated proteins, the proteins corresponding to the column on the underside of transverse axis 0 represent down-regulated proteins, numbers on columns represent multiple differences (nonlogarithmic), for example: If the number in the underside column is 2 , it means that WM is twice as much as IM in expression level; if the number in the upside column is 2, it means that WM is 0.5 times as much as IM in expression level. 

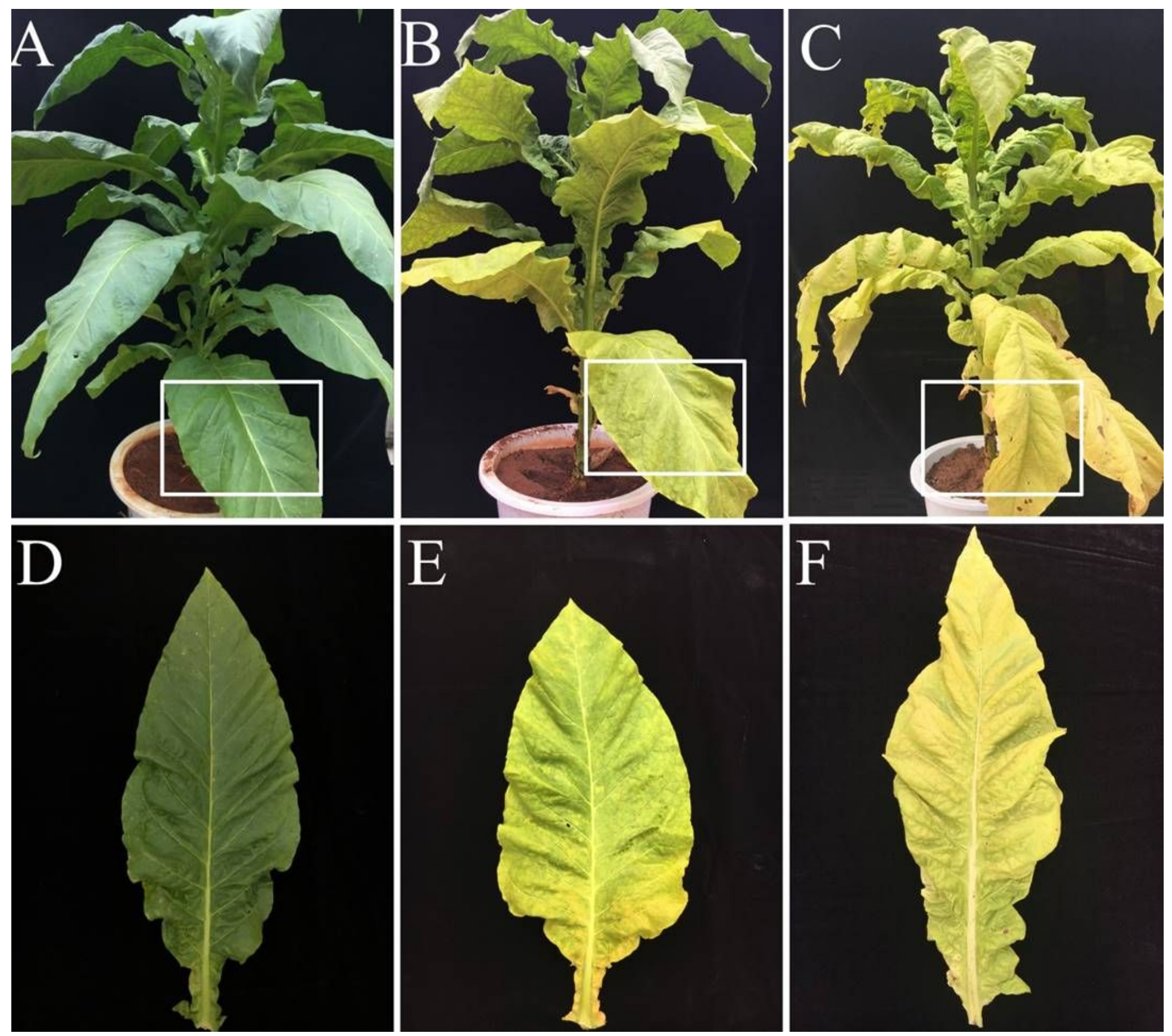

Figure 10

External morphology of tobacco leaves with different maturity Note: (A), (D) IM; (B), (E) WM; (C), (F) OM. 


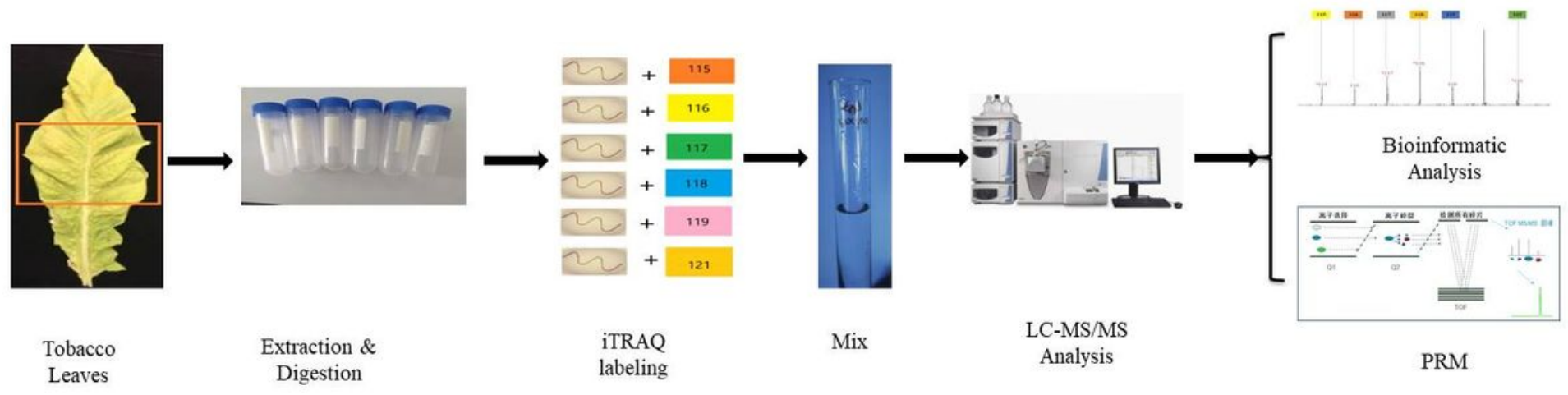

Figure 11

Flow chart of protein separation and identification

\section{Supplementary Files}

This is a list of supplementary files associated with this preprint. Click to download.

- TableS2.xIsx

- TableS1.xlsx

- TableS3.xIsx 